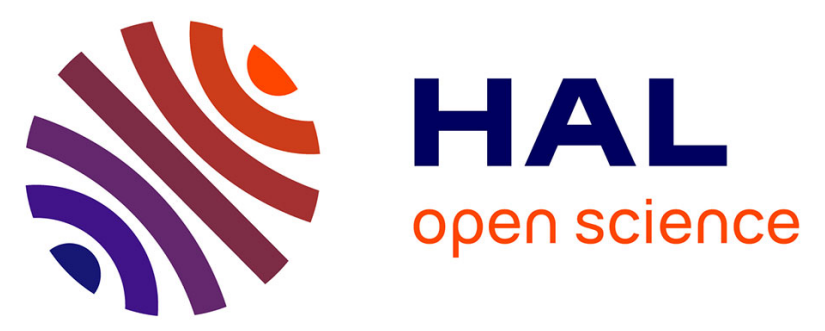

\title{
Les pyroclastites du sud-ouest du lac de Van (Anatolie Orientale, Turquie): Implications dans la paléohydrographie régionale
}

\author{
Damase Mouralis, Catherine Kuzucuoğlu, Ebbru Akköprü, Ali Fuat Dogu, \\ Stéphane Scaillet, Aurélien Christol, Halil Zorer, Daniel Brunstein, Monique \\ Fort, Herve Guillou
}

\section{To cite this version:}

Damase Mouralis, Catherine Kuzucuoğlu, Ebbru Akköprü, Ali Fuat Dogu, Stéphane Scaillet, et al.. Les pyroclastites du sud-ouest du lac de Van (Anatolie Orientale, Turquie): Implications dans la paléohydrographie régionale. Quaternaire, 2010, Biodiversité au quaternaire: climats environnements et peuplements, 21 (4), pp.425 - 441. 10.4000/quaternaire.5739 . hal-02978683

\section{HAL Id: hal-02978683 https://hal.science/hal-02978683}

Submitted on 26 Oct 2020

HAL is a multi-disciplinary open access archive for the deposit and dissemination of scientific research documents, whether they are published or not. The documents may come from teaching and research institutions in France or abroad, or from public or private research centers.
L'archive ouverte pluridisciplinaire HAL, est destinée au dépôt et à la diffusion de documents scientifiques de niveau recherche, publiés ou non, émanant des établissements d'enseignement et de recherche français ou étrangers, des laboratoires publics ou privés. 


\title{
LES PYROCLASTITES DU SUD-OUEST DU LAC DE VAN (ANATOLIE ORIENTALE, TURQUIE): IMPLICATIONS DANS LA PALÉOHYDROGRAPHIE RÉGIONALE
}

\author{
Damase MOURALIS ${ }^{1}$, Catherine KUZUCUOGLU ${ }^{2}$, Ebbru AKKÖPRÜ ${ }^{3}$, \\ Ali Fuat DOGU ${ }^{4}$, Stéphane SCAILLET ${ }^{5}$, Aurélien CHRISTOL ${ }^{2,6}$, Halil ZORER ${ }^{4}$, \\ Daniel BRUNSTEIN ${ }^{2}$, Monique FORT ${ }^{6} \&$ Hervé GUILLOU ${ }^{5}$
}

\begin{abstract}
RÉSUMÉ
Situé en Anatolie orientale, le lac de Van est le quatrième lac fermé du monde par son volume et sa superficie. Sur son pourtour, de nombreuses terrasses témoignent des variations de son niveau au cours du Pléistocène. La partie nord du lac de Van est constituée d'une série de volcans quaternaires ayant émis des pyroclastites dont certaines sont interstratifiées avec ces dépôts lacustres. La présente étude porte sur la partie sud-ouest du lac où l'étude des produits volcaniques en relation stratigraphique avec les dépôts lacustres permet d'établir une téphrostratigraphie régionale dont le calage chronologique $(\mathrm{Ar} / \mathrm{Ar})$ est en cours. Deux ignimbrites successives ont rempli et fossilisé les paléo-vallées, désorganisant totalement le réseau hydrographique régional et séparant progressivement le bassin versant du lac de Van de celui du Tigre. Finalement, l'activité volcanique semble à la fois responsable du caractère endoréique du lac de Van et de la transgression majeure du lac, datée de $c a .100 \mathrm{ka}$. Celle-ci ne serait pas seulement une réponse à des variations du bilan hydrique mais correspondrait d'abord à un forçage d'origine volcanique.
\end{abstract}

Mots-clés: Téphra, Téphrostratigraphie, Paléo-hydrographie, Lac de Van, Anatolie.

\section{ABSTRACT}

THE PYROCLASTITES AROUND SOUTHWESTERN PART OF VAN LAKE (EATERN ANATOLIA, TURQUEY): IMPLICATIONS ON THE REGIONAL PALAEOHYDROGRAPHY

Located in eastern Anatolia (Turkey), Lake Van is the fourth largest lake in the world. Along the banks of the lake, several lake terraces record Pleistocene variations of the lake levels. In the terraces, the lake deposits are often interbedded with pyroclastites emitted by the volcanoes located on the northern side of the lake. Our research focuses on the south-western part of the lake, where several pyroclastites interbedded with lake deposits allow establishing a regional tephrostratigraphy, which is being $\mathrm{Ar} / \mathrm{Ar}$ dated. In this area, the study of the pyroclastites allows to understand the impact of volcanism on the palaeohydrography: all the valleys where filled-in with pyroclastites which induced heavy disruptions of the drainage network. Moreover, our research enlightens evidences that volcanic activity was responsible for the enclosure of the lake and that the major transgression of the lake, dated ca. $100 \mathrm{ka}$, is not only linked to changes in the water balance, but at first linked to volcanic activity.

Key-words: Tephra, Tephrostratigraphy, Palaeo-hydrography, Van Lake, Anatolia.

\section{1 - INTRODUCTION}

Le lac de Van est situé en Anatolie orientale à une altitude de $1648 \mathrm{~m}$. Par sa superficie $\left(>3600 \mathrm{~km}^{2}\right)$ et son volume $\left(>620 \mathrm{~km}^{3}\right)$, il s'agit du quatrième lac fermé au monde. Les nombreux dépôts lacustres qui jalonnent le pourtour du lac témoignent des variations de son niveau au cours du Pléistocène.
Les résultats présentés ici ont été obtenus dans le cadre du programme ANOVAN, débuté en été 2006, qui porte sur l'interprétation climatique et environnementale des variations de niveau du lac de Van en Anatolie orientale, à partir de l'étude détaillée des dépôts lacustres affleurant sur son pourtour (Christol et al., 2008; Christol et al., 2010). Ces dépôts sont parfois interstratifiés avec de nombreux produits pyroclastiques en place (retombées

\footnotetext{
${ }^{1}$ Université de Rouen - CNRS, UMR 6266 (IDEES), rue Lavoisier 76821 Mont-Saint-Aignan. Laboratoire de Géographie Physique,

UMR 8591, CNRS-Université Paris1. 1 place Aristide-Briand, 92195 Meudon cedex. France. Courriel: damase.mouralis@univ-rouen.fr

${ }^{2}$ Laboratoire de Géographie Physique, UMR 8591, CNRS-Université Paris1. 1 place Aristide-Briand, 92195 Meudon cedex. France.

${ }^{3}$ Université d'Istanbul. Département de Géographie. 34452 Beyazit/Eminonu. Istanbul. Turquie.

${ }^{4}$ Université de Van, Département de Géographie. Cografya bölümü. Yüzüncü Yil Universitesi Rektörlügü - 65080 Kampüs - Van. Turquie.

${ }^{5}$ Laboratoire des Sciences de l'Environnement et du Climat, UMR 1572, CEA-CNRS. LSCE-Vallée, Bât. 12, avenue de la Terrasse,

91198 Gif-sur-Yvette cedex. France

${ }^{6}$ Université Paris7, UMR 8586 PRODIG, Equipe DYNMIRIS. UFR GHSS, Case 7001, Université Paris7-Denis Diderot, 2 place Jussieu, 75251 Paris Cedex 05. France.
} 
pliniennes ou écoulements pyroclastiques) qui peuvent servir à la définition du cadre chronologique des variations de niveau du lac, ainsi qu'à caractériser et dater l'activité des volcans ayant pu modifier les caractères hydrologiques et morphologiques de la région (Mouralis et al., 2007; Mouralis et al., 2008).

Le présent article est centré sur la partie sud-ouest du lac, où affleurent de nombreuses pyroclastites, dont certaines sont en connexion stratigraphique avec des dépôts lacustres. Ces pyroclastites (coulées cendroponceuses, ignimbrites, retombées pliniennes) remplissent partiellement des vallées et leur mise en place semble avoir fortement perturbé le réseau hydrographique régional. L'objectif, ici, est de discuter du rôle de l'activité volcanique: i) dans la désorganisation du réseau hydrographique de la partie occidentale du bassin du lac de Van et ii) dans le caractère endoréique de ce bassin lacustre. En effet, nos recherches permettent de penser que les variations pléistocènes du niveau du lac ne sont pas seulement une réponse à des variations du bilan hydrique d'origine climatique mais correspondent aussi à un forçage d'origine volcanique.

\section{2 - PRÉSENTATION DU SECTEUR ÉTUDIÉ}

La partie méridionale du bassin versant correspond au massif de Bitlis, formé de roches métamorphiques, dont les sommets dépassent les 3000 mètres. Si quelques volcans monogéniques sont présents au sud du lac, l'essentiel des appareils volcaniques est localisé le long de la rive nord (fig. 1).

\section{ACTIVITÉ VOLCANIQUE}

$\mathrm{Au}$ nord, une série de volcans quaternaires forme un alignement sud-ouest nord-est, le long duquel se succèdent des volcans composites comme le Nemrut et le Süphan (puis l'Ararat, en dehors du bassin versant du lac de Van), un complexe volcanique rhyolitique (le Meydan) puis un volcan bouclier (Tendürek). Quelques appareils monogéniques isolés sont également présents, à l'est (dôme de Müradiye) et à l'ouest (secteur Dibekli-Incekaya, cf. infra). Tous ces appareils ont été actifs au cours du Quaternaire comme en témoignent les âges radiométriques publiés par Yilmaz, Güner \& Saroğlu (1998) et les nouvelles dates concernant le Nemrut publiées par Çubukçu (2008). En outre, des témoignages d'archives indiquent que le Nemrut a été actif jusqu'à la période historique (Oswald, 1912; Karakhanian et al., 2002; Aydar et al., 2003). Cependant, à l'exception du Nemrut qui a été relativement bien étudié ces dernières années (Aydar et al., 2003; Karaoğlu et al., 2005; Özdemir et al., 2006; Çubukçu, 2008; Ulusoy et al., 2008), les autres volcans sont beaucoup moins connus.

\section{HYDROGRAPHIE}

Le bassin du lac de Van est un bassin endoréique séparé du bassin du Tigre au sud par la chaîne de Bitlis, du bassin de l'Euphrate au nord par le Nemrut et le Süphan, et du bassin de l'Araxe au nord par les massifs volcaniques du Meydan et du Tendürek.

L'étude détaillée des topographies du bassin versant (Christol et al., 2008; Christol et al., 2010) montre trois seuils par lesquels le lac est susceptible de se déverser: i) $1636 \mathrm{~m}(+88 \mathrm{~m})$ dans la vallée du Güzeldere au sud-est du lac; ii) $1765 \mathrm{~m}(+117 \mathrm{~m})$ dans la vallée de Değirmen, au sud du lac; et iii) à $1780 \mathrm{~m}$ (+142 m) à l'ouest du lac, immédiatement au sud du Nemrut, où le seuil est formé par un ensellement séparant le lac de Van de la dépression de Muş et de la vallée de Bitlis. Ces deux premiers seuils topographiques sont situés au fond de larges vallées mal drainées. Cependant aucune morphologie particulière ne révèle leur présence, ni celle d'une ligne de partage des eaux, située perpendiculairement à ces vallées. En outre, les seuils i) et iii) sont situés dans des secteurs remplis par d'épais écoulements pyroclastiques, alors que le seuil ii) est localisé dans un secteur karstique.

Ces observations soulignent la désorganisation d'un réseau hydrographique caractérisé par des vallées remplies par des pyroclastites, et parfois drainées dans des directions divergentes de part et d'autre de seuils topographiques peu ou pas marqués dans les paysages. Ces seuils, pourtant, isolent le lac terminal des bassins exoréiques voisins (Tigre et Euphrate).

\section{VARIATIONS PLÉISTOCÈNES DU NIVEAU DU LAC}

Les terrasses qui jalonnent les rives du lac de Van témoignent des fortes amplitudes des variations récentes du niveau du lac. Valeton (1978) puis Kempe et al. (2002) ont présenté de premières analyses des dépôts constituant ces terrasses. Une étude systématique des terrasses lacustres est actuellement réalisée dans le cadre du programme ANOVAN. Les résultats en cours de publication présentent et interprètent de façon détaillée les enregistrements des terrasses lacustres et les niveaux du lac dont elles témoignent (Christol et al., 2008; Christol et al., 2010; Kuzucuoğlu et al., 2010).

La partie orientale du lac présente les terrasses lacustres les mieux développées: ces terrasses ont été regroupées en quatre niveaux principaux, qui correspondent à des enregistrements de transgressions suivies de régressions présentant des paliers. D'après Christol et al. (2010) et Kuzucuoğlu et al. (2010) les séquences sédimentaires composant les quatre groupes de terrasses (T1 à T4) permettent d'identifier et reconstituer au moins deux cycles de transgression-régression $(\mathrm{C} 1$ et C2) pendant lesquels le lac a dépassé son niveau actuel et a ennoyé les basses vallées des affluents du lac où se sont accumulés les dépôts lacustres correspondants. Les deux terrasses les plus élevées appartiennent au cycle 1 (C1) et sont respectivement situées à 1745/55 m (T1, ca $+100 / 110 \mathrm{~m}$ par rapport au niveau actuel du lac) et à 1715/m (T2, $c a+70 / 85 \mathrm{~m})$. Si l'altitude de certaines terrasses appartenant au groupe T1 apparaît avoir été déterminée partiellement par un soulèvement tectonique (vallée de la Karasu au nord de Van), certains témoins de $\mathrm{T} 1$, non déformés, correspondent à des dépôts de lac profond, signalant une toute première transgression 

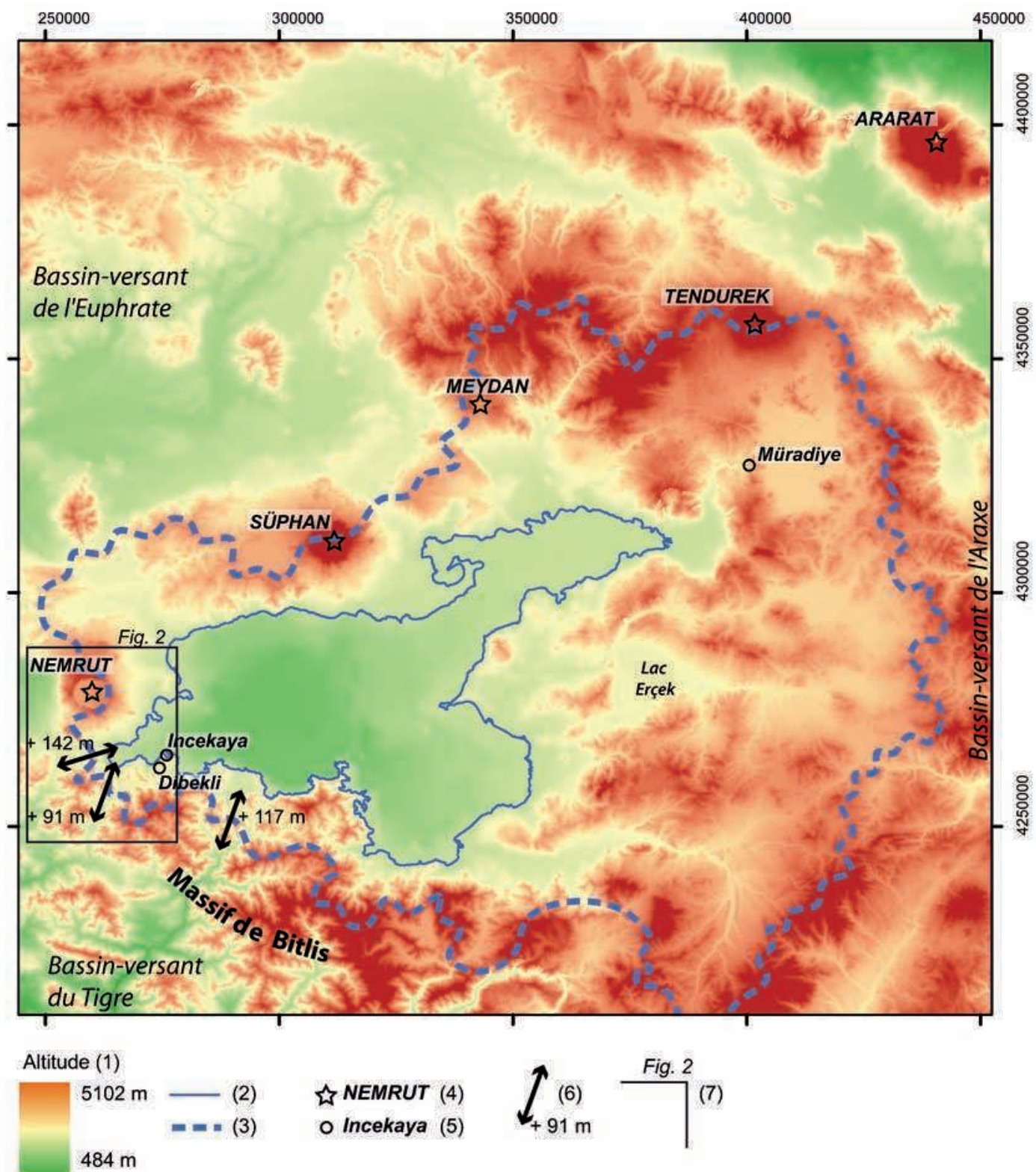

Fig. 1: Localisation du lac de Van.

1- Altitudes issues des données SRTM et bathymétrie issue de Degens \& Kurtmann (1978), compilés par Christol et al. (2008). 2- Niveau actuel du lac (1 $647 \mathrm{~m})$. 3- Limite du bassin versant. 4- Volcans composites. 5- Volcans monogéniques. 6- Seuils de déversements potentiels du lac et altitudes relatives au-dessus du niveau actuel du lac. 7- Localisation de la figure 2.

Fig. 1: Location of Van Lake. 1 - Elevation (SRTM Data) - Bathymetry from Degens \& Kurtmann (1978), compiled by Christol et al. (2008). 2- Present day lake level $(1647 \mathrm{~m})$. 3- Limit of Lake Van watershed. 4-Composite volcanoes. 5- Monogenic volcanoes. 6- Potential topographic thresholds of the lake and their relative elevations above present lake level. 7-Location of fig. 2

(C1') particulièrement importante $(c a>1770 \mathrm{~m}, c a$ $+125 \mathrm{~m}$ ) (Kuzucuoğlu et al., 2010). Après ces deux hauts niveaux lacustres initiaux ( $\mathrm{C} 1$ ' et $\mathrm{C} 1$ " responsables du dépôt des terrasses T1 non déformée et T2), une forte incision fluviatile témoigne d'une importante régression. Ensuite, une nouvelle transgression (C2) est responsable du dépôt de sédiments lacustres formant deux nouveaux groupes de terrasses; le premier groupe, à $c a+50 / 40 \mathrm{~m}$ (T3) correspond à la transgression initiale du cycle $\mathrm{C} 2$ en deux remontées successives (C2' et $\mathrm{C} 2$ ") ; le deuxième groupe à +10 à $+25 \mathrm{~m}$ (T4) correspond à des terrasses d'érosion dans les dépôts lacustres et à d'anciennes terrasses alluviales exhumées. Il semble en outre que certaines terrasses dont le sommet atteint $+10 / 12 \mathrm{~m}$ enregistrent une dernière remontée du niveau (C3 ?) qui aurait succédé à la très forte baisse du niveau terminant le cycle C2. Pour une présentation détaillée des terrasses et de leur interprétation en terme de variations du niveau du lac, voir Kuzucuoğlu et al., (2010) et Christol et al. (2010)

Les terrasses T2 situées à $+80 / 85 \mathrm{~m}$, correspondent à un niveau du lac vers 1730/35 m (transgression C1"). Un tel niveau témoigne d'un volume d'eau de $960 \mathrm{~km}^{3}$, soit près de $300 \mathrm{~km}^{3}$ de plus qu'aujourd'hui (Christol et al., 2010).

Une telle transgression peut s'expliquer par: i) une modification brutale du bilan hydrique et/ou ii) la fermeture d'un ancien exutoire, en relation avec l'activité volcanique et/ou la tectonique régionale. 


\section{LE SUD-OUEST DU LAC DE VAN}

Localisé au sud-ouest du lac de Van, le secteur étudié dans le présent article est composé de trois vallées (Küçüksu, Kotum et Güzeldere), en partie remplies par des pyroclastites (fig. 2 et 3 ). La vallée du Küçüksu est drainée vers l'ouest, par une rivière qui, au-delà du village de Küçüksu, décrit un coude et emprunte alors la vallée de Kotum, drainée vers le lac au nord. Dans la vallée de Kotum, la rivière incise les pyroclastites de «l'ignimbrite de Kotum», des travertins et des dépôts lacustres (bottomsets, cône deltaïque). Une troisième vallée, celle du Güzeldere est orientée nord-est-sud-ouest. Elle est drainée de manière divergente. Au nord, l'écoulement rejoint la rivière qui draine successivement les vallées de Küçüksu et de Kotum; alors qu'au sud, au-delà d'une ligne de partage des eaux située perpendiculairement à la vallée, l'écoulement se fait en direction du Tigre. Cette ligne de partage des eaux qui limite le bassin endoréique de Van de celui du Tigre est située à environ $1736 \mathrm{~m}(+88 \mathrm{~m})$

Enfin, à l'amont de la vallée du Küçüksu, le secteur de Dibekli-Incekaya comporte des édifices volcaniques monogéniques à l'origine de certaines pyroclastites présentes dans les trois vallées décrites précédemment. On y observe un tuff-cone (Incekaya) dont le cratère est ouvert sur le lac et plusieurs cônes stromboliens (à proximité du village de Dibekli).

Dans ces vallées, des replats topographiques suggèrent l'existence de terrasses. Les vallées du Küçüksu comme

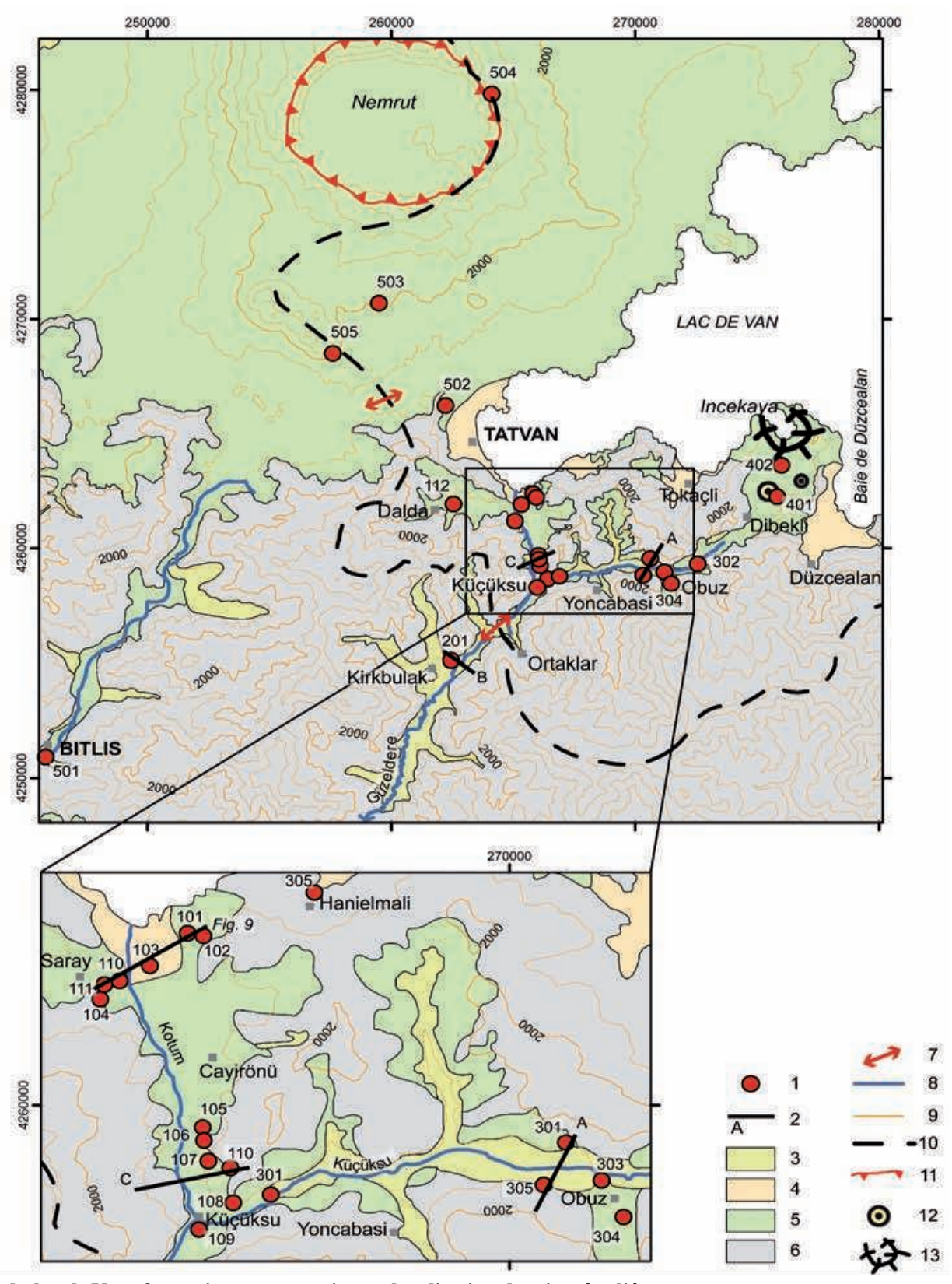

Fig. 2 : Le sud-ouest du lac de Van : formations quaternaires et localisation des sites étudiés.

1- Localisation des sites étudiés (cf. figure 8). 2- Localisation des coupes des figures 3 et 9. 3- Alluvions. 4- Formations lacustres. 5- Pyroclastites (indifférenciées). 6- Substratum (roches métamorphiques du massif de Bitlis). 7- Seuils actuels de déversement potentiel du lac. 8- Réseau hydrographique. 9- Courbes de niveau (équidistance: $200 \mathrm{~m}$; données SRTM) 10- Limites des bassins-versants du lac de Van et du Tigre. 11- Caldeira du Nemrut. 12- Cônes de scories. 13- Cône de tuf.

Fig. 2: The south-western part of Van Lake: Quaternary deposits and location of studied sections. 1- Location of sections studied (see fig. 8). 2- Location of sections (figures 3 and 9). 3-Alluvium. 4- Lake deposits. 5- Pyroclastites (un-differenciated). 6- Substratum (Bitlis metamorphics). 7- Present topographic thresholds. 8-River network. 9-Elevation curves (200 m; SRTM Data). 10-Border line between drainage areas of Lake Van and Tigris River. 11-Nemrut Caldera. 12- Scoria cones. 13-Tuff cone. 


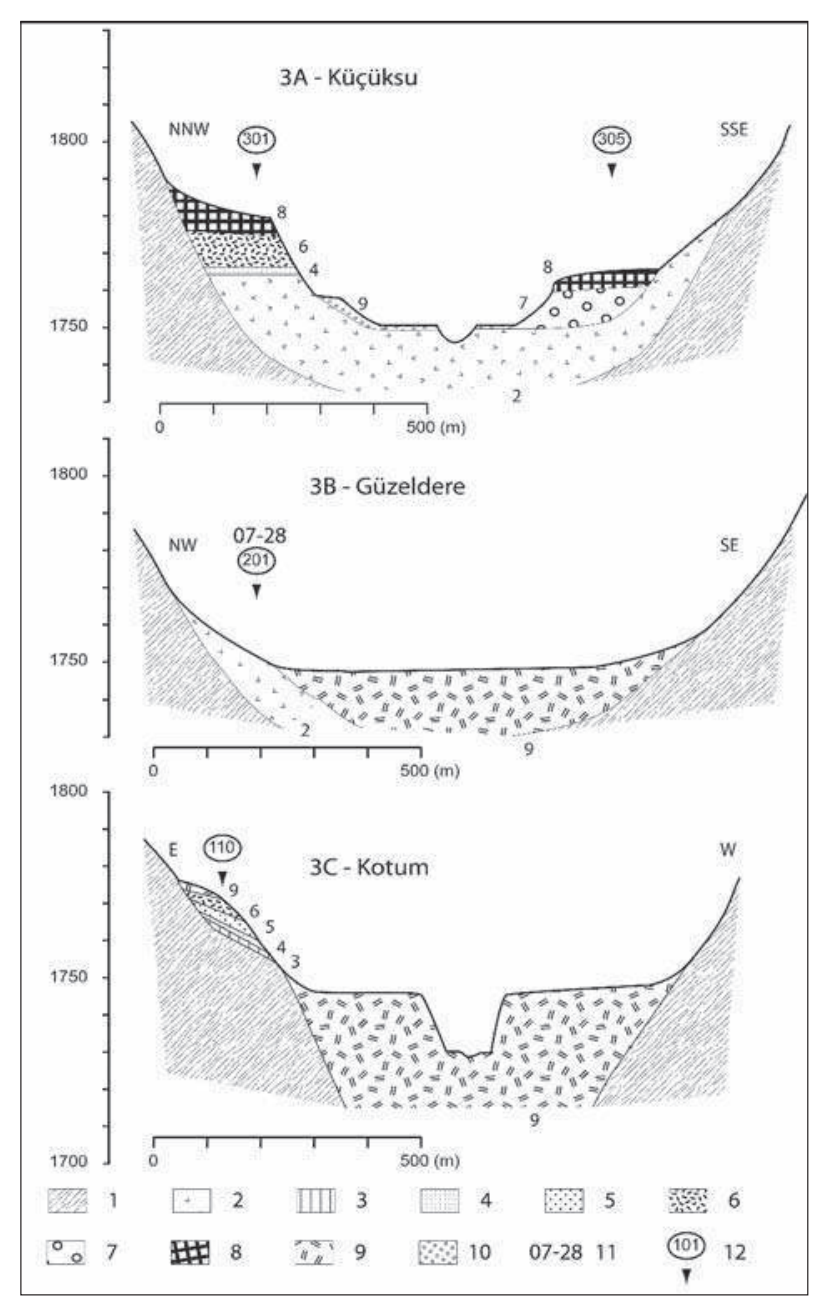

Fig. 3 : Coupes à travers les trois vallées de Güzeldere (A), Küçüksu (B) et de Kotum (C).

Cf. figure 2 pour la localisation des profils.

1- Substrat métamorphique. 2- Ignimbrite d'Obuz (PYR-01). 3Paléosol sur colluvions. 4- Retombée cendreuse (PYR-02). 5- Coulée cendro-ponceuse (PYR-02). 6- Retombée ponceuse (PYR-02). 7- Alluvions grossières. 8- Tuf lité noir d'Incekaya (PYR-03). 9- Ignimbrite de Kotum (PYR-05). 10- Alluvions. 11- Numéro d'échantillon. 12- Localisation des sites (cf. fig. 2).

Fig. 3: Section across the three valleys: (A) Güzeldere, (B) Küçüksu and (C) Kotum. See fig. 2 for the location of these sections. 1-Metamorphic rocks. 2- "Obuz ignimbrite" (PYR-01). 3-Paleosoil topping colluvium. 4-Ash fall (PYR-02). 5- Ash and pumice flow (PYR-02). 6-Pumice fall (PYR-02). 7- Coarse alluvium. 8- Incekaya black tuff (PYR-03). 9Kotum ignimbrite (PYR-05). 10-Alluvium. 11-Sample name. 12-Site location (see fig. 2).

du Güzeldere présentent un fond plat où un petit cours d'eau sous-calibré par rapport au gabarit des vallées décrit des méandres. Dans la vallée de la Küçüksu (fig. $3 \mathrm{~A}$ ), large de 150 à $250 \mathrm{~m}$, les versants comportent un replat situé à $c a+30 \mathrm{~m}$, suffisamment continu pour qu'un petit canal d'irrigation, probablement ancien mais toujours fonctionnel, y ait été installé.

La vallée du Güzeldere (fig. 3B), dont le fond plat dépasse parfois une largeur de $500 \mathrm{~m}$, est largement surcalibrée par rapport à l'écoulement actuel, quasi nul. Dans cette vallée, le seuil séparant les bassins versants du lac de Van et du Tigre n'est absolument pas marqué dans la topographie car, en amont comme en aval de ce seuil, la vallée présente les mêmes morphologies. On note que l'écoulement s'y effectue difficilement, en partie en raison du développement d'un travertin toujours actif (Kükürtlü pınar) en amont duquel des zones hydromorphes se sont développées. Vers le sud, au-delà de ce travertin, la vallée se resserre, sa pente augmente, et le Güzeldere s'engouffre dans des gorges en direction du Tigre.

A proximité du village de Küçüksu, la vallée de Kotum (fig. 3C) n'est large que d'une centaine de mètres, puis elle s'ouvre en direction du lac jusqu'à déboucher sur le delta récent de la rivière.

Les versants des gorges de la vallée de Kotum présentent trois replats. Le premier, situé à $c a+30 / 40 \mathrm{~m}$ nivelle d'amont en aval l'ignimbrite de Kotum, des travertins puis des dépôts lacustres. Sous ce replat, la rivière Kotum a incisé en gorge ces trois formations, décrivant des méandres encaissés. Le second replat, situé à $+70 / 80 \mathrm{~m}$ environ, est incliné vers le centre de la vallée et présente des dépôts lacustres (fan delta) interstratifiés avec des pyroclastites (retombée plinienne). Un troisième replat est situé à $c a+90 / 100 \mathrm{~m}$ et il se raccorde vers l'amont de la vallée au replat observé dans la vallée du Küçüksu. Dans ces deux vallées, ce replat est inscrit dans un tuf noir lité, de granulométrie souvent homogène et fine.

\section{3 - MÉTHODOLOGIE}

\section{TÉPHROSTRATIGRAPHIE}

Les reconstitutions stratigraphiques et morphologiques reposent sur la reconnaissance et la caractérisation des produits volcaniques utilisés comme horizons-repères pour établir des corrélations longue distance. La caractérisation de ces pyroclastites s'effectue grâce à la reconnaissance macroscopique: i) des faciès de ponces, plus ou moins vésiculées; ii) des assemblages minéralogiques (porphyrisme), ainsi que iii) des lithiques associés. Le tableau 1 présente les analyses effectuées pour chaque échantillon, ainsi que les sites où ils ont été prélevés (localisés sur la figure 2).

\section{GÉOCHIMIE DES PYROCLASTITES}

Vingt-trois échantillons provenant d'écoulements cendro-ponceux plus ou moins indurés et de retombées ponceuses ont fait l'objet d'analyses géochimiques ICP-AES sur roche totale au Service d'analyse des roches et minéraux (SARM, Nancy) du CNRS. Dans la mesure du possible, ces analyses ont porté sur les produits vésiculés, afin d'étudier la composition chimique des matériaux juvéniles. Les résultats analytiques sont donnés dans le tableau 2.

Replacé sur un diagramme TAS (fig. 4), ces produits différenciés appartiennent aux champs des trachytes et des rhyolites. La figure 4 ainsi que les différents diagrammes binaires qui croisent les teneurs en $\mathrm{SiO} 2$ et divers oxydes (fig. 5) permettent de distinguer trois groupes principaux :

i) Les produits les moins différenciés présentent des teneurs en $\mathrm{SiO}_{2}$ comprises entre 62 et $64 \%$ et regroupent une série d'échantillons prélevés sur les flancs ou à proximité du Nemrut. 


\begin{tabular}{|c|c|c|c|c|}
\hline $\begin{array}{c}\text { Ensemble } \\
\text { pyroclastique }\end{array}$ & Site & Echantillon & Analyse & Description \\
\hline PYR-01 & 302 & 07-01 & ICP-AES & Ignimbrite noire, non indurée à grosses ponces porphyriques et enclaves basaltiques \\
\hline PYR-01 & 302 & 07-02 & ICP-AES & Ignimbrite noire, indurée à grosses ponces porphyriques, fiammes, et enclaves basaltiques \\
\hline PYR-01 & 201 & 07-28 & ICP-AES & Ignimbrite noire, non indurée, à grosses ponces porphyriques et enclaves basaltiques \\
\hline PYR-05 & 110 & 07-04 & ICP-AES & Ignimbrite grise, indurée, rares ponces, fiammes, enclaves \\
\hline PYR-05 & 110 & 07-05 & ICP-AES & Ignimbrite grise, non indurée, rares ponces et petites enclaves \\
\hline PYR-05 & 110 & $07-06$ & ICP-AES & Ignimbrite grise, indurée, rares ponces, fiammes, enclaves \\
\hline PYR-05 & 110 & 07-07 & ICP-AES & Ignimbrite grise, indurée, rares ponces, fiammes, enclaves \\
\hline PYR-05 & 110 & 07-09 & ICP-AES & Ignimbrite grise, non indurée, rares ponces et petites enclaves \\
\hline PYR-05 & 111 & 07-12 & ICP-AES & Ignimbrite grise, au sommet du travertin \\
\hline PYR-05 & 103 & $07-21$ & ICP-AES & Ignimbrite rosâtre, non indurée \\
\hline PYR-05 & 109 & $07-29$ & ICP-AES & Ignimbrite grise, non indurée, rares ponces et petites enclaves \\
\hline PYR-05 & 109 & $07-30$ & ICP-AES & Ignimbrite grise, indurée, rares ponces, fiammes, enclaves \\
\hline PYR-05 & 107 & $07-31$ & ICP-AES & Ignimbrite grise, non indurée, rares ponces et petites enclaves \\
\hline PYR-05 & 112 & $07-20$ & ICP-AES & Ignimbrite grise, indurée, rares ponces, fiammes, enclaves \\
\hline$?$ & 111 & $07-10$ & ICP-AES & Ignimbrite gise, emboîtées dans les travertin \\
\hline Nemrut & 501 & $07-46$ & ICP-AES & Ignimbrite rougeâtre à noire, indurée. Vallée Bitlis \\
\hline Nemrut & 305 & $07-18$ & ICP-AES & Ignimbrite grise. Hanielmali \\
\hline Nemrut & 502 & $07-48$ & ICP-AES & Ignimbrite rougeâtre. NE Tatvan. \\
\hline Nemrut & 503 & $07-34$ & ICP-AES & Ignimbrite rougeâtre à noire. Nemrut, route sud \\
\hline Nemrut & 504 & $07-41$ & ICP-AES & Retombée plinienne. Ponces porphyriques. Sommet du Nemrut \\
\hline Nemrut & 504 & $07-42$ & ICP-AES & Retombée plinienne. Ponces porphyriques. Sommet du Nemrut \\
\hline Nemrut & 505 & $07-43$ & ICP-AES & Lave. Dôme de Kirkör \\
\hline Süphan & - & 034 & WDS & Ponce du Süphan (Mollafadil) \\
\hline Süphan & - & 032 & WDS & Ponce du Süphan (nord Adilcevaz) \\
\hline Süphan & - & 041 & WDS & Ponce du Süphan (sud Patnos) \\
\hline Nemrut & 504 & 003 & WDS & Ponces du Nemrut \\
\hline PYR-01 & 302 & 002 & WDS & Ponces de lignimbrite noire, porphyriques \\
\hline PYR-02 & 101 & 021 & WDS, Ar/Ar & Ponces du Nemrut, porphyriques \\
\hline - & - & 030 & WDS, Ar/Ar & Retombée ponceuse. Ponces prophyriques \\
\hline
\end{tabular}

Tab. 1 : Localisation des échantillons.

Cf. figure 2 pour la localisation des sites, et figures 4, 5, 6 et 7 pour les résultats des analyses.

Tab. 1: Samples locations. Cf. figure 2 for the location of studied sites and figure 4, 5, 6 and 7 for the analytical results.

\begin{tabular}{|c|c|c|c|c|c|c|c|c|c|c|c|c|}
\hline Echantillon & $\begin{array}{c}\mathrm{SiO2} \\
\%\end{array}$ & $\begin{array}{c}\mathrm{Al} 2 \mathrm{O} 3 \\
\%\end{array}$ & $\begin{array}{c}\mathrm{Fe} 2 \mathrm{O} 3 \\
\%\end{array}$ & $\begin{array}{c}\mathrm{MnO} \\
\% \\
\end{array}$ & $\begin{array}{c}\mathrm{MgO} \\
\%\end{array}$ & $\begin{array}{c}\mathrm{CaO} \\
\%\end{array}$ & $\begin{array}{c}\mathrm{Na2O} \\
\%\end{array}$ & $\begin{array}{c}\text { K2O } \\
\%\end{array}$ & $\begin{array}{c}\text { TiO2 } \\
\%\end{array}$ & $\begin{array}{c}\text { P2O5 } \\
\%\end{array}$ & $\begin{array}{l}\text { PF } \\
\% \\
\end{array}$ & $\begin{array}{c}\text { Total } \\
\%\end{array}$ \\
\hline 07-01 & 66.00 & 14.48 & 4.05 & 0.11 & 0.06 & 1.15 & 4.05 & 5.56 & 0.30 & 0.04 & 3.32 & 99.13 \\
\hline $07-02$ & 64.65 & 15.06 & 4.98 & 0.13 & 0.20 & 1.59 & 4.19 & 4.80 & 0.43 & 0.08 & 2.51 & 98.63 \\
\hline $07-04$ & 70.32 & 10.94 & 4.39 & 0.09 & 0.04 & 0.35 & 5.01 & 4.40 & 0.27 & 0.03 & 2.67 & 98.52 \\
\hline 07-05 & 69.23 & 11.60 & 4.65 & 0.10 & 0.07 & 0.39 & 5.01 & 4.19 & 0.29 & 0.03 & 3.15 & 98.72 \\
\hline $07-06$ & 70.26 & 10.89 & 4.59 & 0.10 & 0.07 & 0.48 & 5.15 & 4.39 & 0.28 & 0.04 & 2.51 & 98.74 \\
\hline $07-07$ & 70.50 & 10.87 & 4.49 & 0.10 & 0.03 & 0.35 & 5.17 & 4.45 & 0.26 & 0.03 & 2.64 & 98.88 \\
\hline 07-09 & 69.72 & 11.28 & 4.63 & 0.10 & 0.07 & 0.41 & 5.07 & 4.24 & 0.27 & 0.03 & 3.39 & 99.22 \\
\hline $07-10$ & 62.46 & 15.35 & 4.85 & 0.07 & 0.12 & 1.03 & 3.61 & 4.67 & 0.36 & 0.06 & 6.02 & 98.59 \\
\hline $07-12$ & 70.51 & 11.02 & 4.56 & 0.11 & 0.05 & 0.39 & 5.11 & 4.46 & 0.27 & 0.03 & 2.79 & 99.29 \\
\hline $07-18$ & 61.48 & 13.99 & 4.30 & 0.12 & 0.29 & 1.85 & 3.37 & 4.49 & 0.34 & 0.06 & 8.50 & 98.79 \\
\hline $07-20$ & 69.90 & 11.19 & 4.61 & 0.11 & 0.06 & 0.45 & 4.64 & 4.19 & 0.28 & 0.03 & 3.27 & 98.72 \\
\hline $07-21$ & 70.15 & 10.66 & 4.55 & 0.11 & < L.D. & 0.34 & 4.86 & 4.56 & 0.25 & 0.03 & 3.54 & 99.04 \\
\hline $07-28$ & 64.31 & 14.86 & 4.92 & 0.13 & 0.14 & 1.43 & 4.04 & 4.87 & 0.38 & 0.06 & 3.47 & 98.61 \\
\hline $07-29$ & 66.80 & 11.86 & 5.24 & 0.10 & 0.18 & 0.44 & 4.16 & 3.67 & 0.27 & 0.03 & 5.77 & 98.53 \\
\hline $07-30$ & 70.41 & 11.45 & 4.68 & 0.11 & 0.05 & 0.41 & 4.68 & 4.18 & 0.27 & 0.04 & 2.79 & 99.07 \\
\hline $07-31$ & 70.94 & 11.05 & 4.66 & 0.10 & 0.05 & 0.45 & 5.09 & 4.57 & 0.28 & 0.03 & 2.99 & 100.20 \\
\hline $07-34$ & 63.16 & 17.70 & 4.51 & 0.14 & 0.33 & 2.43 & 6.09 & 4.02 & 0.50 & 0.12 & 0.79 & 99.79 \\
\hline $07-41$ & 62.14 & 16.61 & 5.05 & 0.12 & 0.74 & 2.73 & 4.81 & 3.40 & 0.71 & 0.21 & 2.05 & 98.57 \\
\hline $07-42$ & 61.98 & 16.12 & 5.37 & 0.12 & 0.94 & 2.71 & 4.82 & 3.47 & 0.75 & 0.21 & 2.10 & 98.59 \\
\hline $07-43$ & 70.91 & 14.15 & 3.48 & 0.08 & $<$ L.D. & 0.60 & 5.37 & 5.05 & 0.24 & 0.03 & 0.19 & 100.08 \\
\hline $07-46$ & 65.20 & 15.37 & 4.39 & 0.11 & 0.16 & 1.55 & 4.64 & 5.33 & 0.44 & 0.09 & 2.61 & 99.90 \\
\hline $07-48$ & 63.08 & 17.48 & 4.36 & 0.12 & 0.41 & 1.71 & 6.50 & 4.75 & 0.51 & 0.12 & 0.21 & 99.23 \\
\hline $07-49$ & 66.90 & 14.58 & 2.29 & 0.08 & 0.70 & 1.49 & 3.19 & 5.12 & 0.56 & 0.16 & 3.50 & 98.56 \\
\hline
\end{tabular}

Tab. 2 : Résultats des analyses géochimiques sur roche totale.

Analyses géochimiques réalisées au SARM (CNRS, Nancy, France) - Eléments majeurs dosés par ICP-AES. Cf. fig. 4 et 5.

Tab. 2: Results of the wool rock geochemical analyses. Geochemical analyses processed in SARM (CNRS, Nancy, France) - Major elements analyzed by ICP-AES. See fig. 4 and 5. 


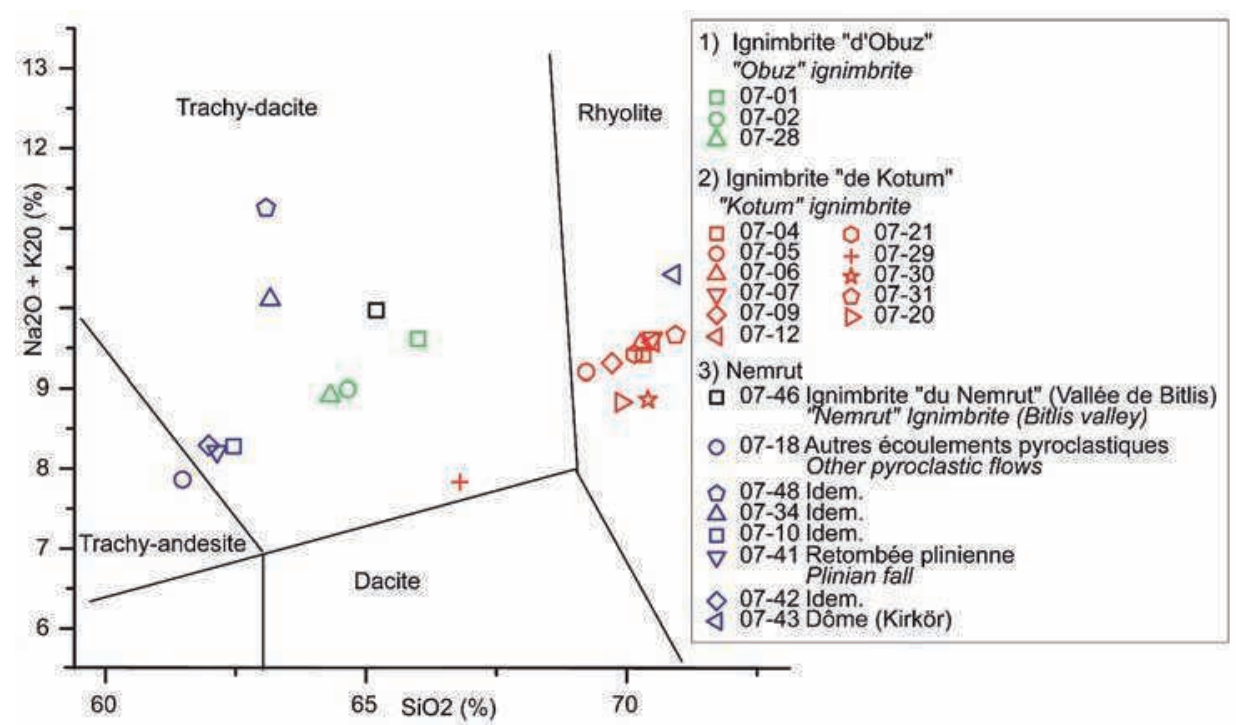

Fig. 4 : Diagramme TAS (d'après Le Maitre, 2002).

Analyses géochimiques réalisées au SARM (CNRS, Nancy, France) - Eléments majeurs dosés par ICP-AES.

Fig. 4: TAS Diagram (according to Lemaitre, 2002). Geochemical analyses processed in SARM (CNRS, Nancy, France) - Major elements analyzed by ICP-AES.

ii) Les échantillons ayant une teneur en $\mathrm{SiO}_{2}$ comprise entre 64 et $66 \%$ correspondent à une ignimbrite de couleur noire («ignimbrite d'Obuz», PYR-01), prélevée dans les vallées du Güzeldere et de la Küçüksu. L'échantillon 07-46, provenant de l'ignimbrite remplissant la vallée de Bitlis, présente une composition chimique proche des échantillons de ce groupe.

iii) Les échantillons ayant une teneur en $\mathrm{SiO}_{2}$ comprise entre 69 et $71 \%$ ont été prélevés dans la vallée de Kotum et proviennent des différents faciès d'une même ignimbrite ( «ignimbrite de Kotum» PYR-05).

Six échantillons ont également fait l'objet d'analyses géochimiques à l'aide d'une microsonde Cameca SX-100 au Service Camparis (Jussieu). Les figures 6A et $\mathrm{B}$ montrent les premiers résultats de ces analyses. Nous avons comparé la géochimie des éléments majeurs des verres et des feldspaths de ponces provenant du Nemrut et du Süphan, qui sont les deux volcans composites les plus proches de notre secteur d'étude ayant émis des produits pliniens. La figure 6A montre que les verres analysés du Süphan forment un groupe très homogène, alors que les verres provenant d'échantillons du Nemrut ont une composition plus variée quoique toujours rhyolitique. Les résultats analytiques sont donnés dans le tableau 3. La figure $6 \mathrm{~B}$ présente la composition des feldspaths de trois pyroclastites, provenant également du Nermrut et du Süphan.

\section{TÉPHROCHRONOLOGIE}

Le calage chronologique repose sur les datations radiométriques. En particulier, 10 dates $\mathrm{Ar} / \mathrm{Ar}$ sont en cours de réalisation au Laboratoire des Sciences du Climat et de l'Environnement (UMR 1572). Les feldspaths nécessaires à la datation sont sélectionnés à l'aide d'une loupe binoculaire et nettoyés dans une solution à $5 \% \mathrm{HF}$ placée dans une cuve à ultrasons, puis soigneusement rincés. Les échantillons sont ensuite irradiés pendant 20 minutes dans le réacteur Osiris (CEA-Saclay, France). Les grains sont ensuite placés dans les trous d'un porteéchantillon (10 à 20 cristaux remplissant 8 trous pour l'échantillon VAN-021) et dégazés en deux étapes à l'aide d'un rayon laser (20 $\mathrm{W} \mathrm{CO} 2)$ : d'abord à basse température, puis à $20 \%$ de la puissance totale du rayon. Ils sont ensuite purifiés avant l'admission dans un spectromètre de masse GV 5400. L'analyse des résultats suit les procédures standards décrites en détail par Scaillet (1996, 2000).

Pour l'instant, deux résultats ont été obtenus. Le premier (fig. 7A), provient de la vallée de Kotum: les feldspaths de l'échantillon VAN-021 ont livré un âge Ar/ Ar de $117 \pm 5,2 \mathrm{ka}$. Ce résultat est en accord avec les âges U/Th publiés par Kuzucuoğlu et al. (2010) concernant des échantillons de travertin prélevés dans le secteur aval de la vallée de Kotum ( ca $110 \mathrm{ka}$ ). Les travertins comme les feldspaths des ponces de l'échantillon VAN-021 sont stratigraphiquement situés sous les dépôts de la transgression lacustre enregistrée dans la vallée de Kotum (cycle 1); travertins et ponces lui sont donc antérieurs. Le second échantillon (fig. 7B) est situé en dehors du secteur étudié dans cet article: l'échantillon VAN-030, provenant de la partie oriental du lac, a livré un âge $\mathrm{Ar}$ / Ar de 31,4 \pm 6,6 ka, datant les dépôts transgressifs du cycle 2 .

\section{ALTITUDES}

Les altitudes indiquées dans cet article proviennent des cartes topographiques au $1: 25000$ et des données SRTM (en particulier pour les figures 1 et 2). En 2007, des mesures réalisées en utilisant un GPS différentiel (équipement RTK-Thalès 6502, composé d'une station de base bi-fréquence et d'un appareil mobile), ont permis de préciser les altitudes des contacts entre les dépôts lacustres et les pyroclastites qu'ils recouvrent dans le secteur de la carrière de Kotum, ainsi que l'altitude du seuil du Güzeldere. Le protocole utilisé et 


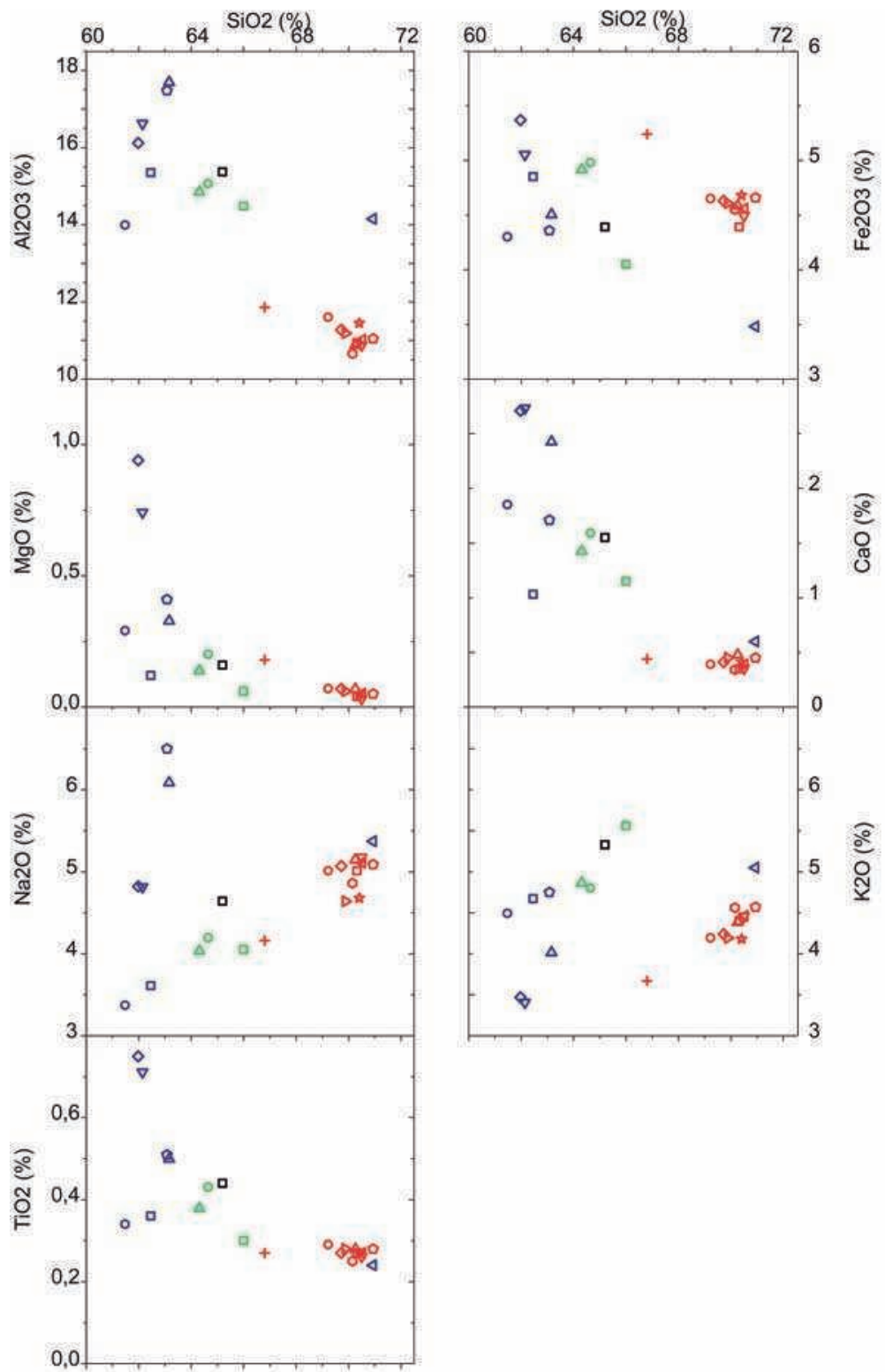

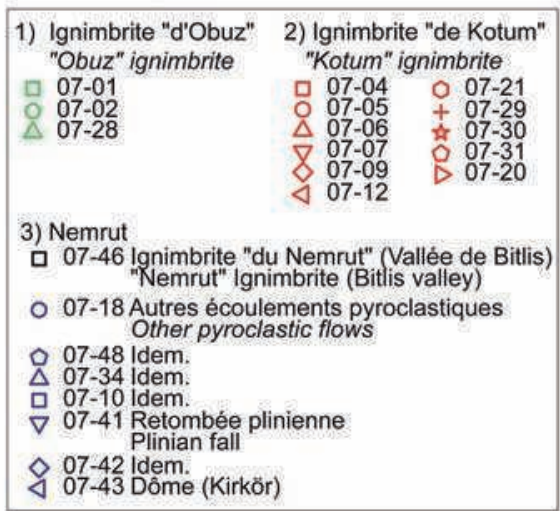

Fig. 5 : Géochimie de quelques pyroclastites du SW du lac de VAN.

Analyses géochimiques réalisées au SARM (CNRS, Nancy, France) - Eléments majeurs dosés par ICP-AES.

Fig. 5: Geochemistry of some pyroclastites from the south-western part of Van Lake

Geochemical analyses processed in SARM (CNRS, Nancy, France) - Major elements analyzed by ICP-AES.

\begin{tabular}{|c|c|c|c|c|c|c|c|c|c|c|c|c|}
\hline SAMPLE & ANAL(1) & $\begin{array}{c}\mathrm{Na} 2 \mathrm{O} \\
(\%)\end{array}$ & $\begin{array}{l}\mathrm{SiO2} \\
(\%)\end{array}$ & $\begin{array}{c}\mathrm{Al} 2 \mathrm{O} 3 \\
(\%)\end{array}$ & $\begin{array}{c}\mathrm{MgO} \\
(\%)\end{array}$ & $\begin{array}{l}\mathrm{K} 2 \mathrm{O} \\
(\%)\end{array}$ & $\begin{array}{c}\mathrm{Cl} \\
(\%)\end{array}$ & $\begin{array}{l}\mathrm{CaO} \\
(\%)\end{array}$ & $\begin{array}{c}\mathrm{TiO} 2 \\
(\%)\end{array}$ & $\begin{array}{c}\mathrm{MnO} \\
(\%)\end{array}$ & $\begin{array}{l}\mathrm{FeO} \\
(\%)\end{array}$ & Total \\
\hline 002 & 9 & $\begin{array}{l}3.87 \\
1.56\end{array}$ & $\begin{array}{r}72.83 \\
3.54\end{array}$ & $\begin{array}{r}14.20 \\
1.86\end{array}$ & $\begin{array}{l}\mathbf{0 . 1 2} \\
0.22\end{array}$ & $\begin{array}{l}4.44 \\
0.80\end{array}$ & $\begin{array}{l}\mathbf{0 . 1 0} \\
0.09\end{array}$ & $\begin{array}{l}0.81 \\
0.77\end{array}$ & $\begin{array}{l}0.23 \\
0.28\end{array}$ & $\begin{array}{l}\mathbf{0 . 1 0} \\
0.07\end{array}$ & $\begin{array}{l}3.20 \\
1.55\end{array}$ & $\begin{array}{r}100 \\
0.15\end{array}$ \\
\hline 003 & 9 & $\begin{array}{l}3.87 \\
1.56\end{array}$ & $\begin{array}{r}72.83 \\
3.54\end{array}$ & $\begin{array}{r}14.20 \\
1.86\end{array}$ & $\begin{array}{l}\mathbf{0 . 1 2} \\
0.22\end{array}$ & $\begin{array}{l}4.44 \\
0.80\end{array}$ & $\begin{array}{l}\mathbf{0 . 1 0} \\
0.09\end{array}$ & $\begin{array}{l}\mathbf{0 . 8 1} \\
0.77\end{array}$ & $\begin{array}{l}\mathbf{0 . 2 3} \\
0.28\end{array}$ & $\begin{array}{l}\mathbf{0 . 1 0} \\
0.07\end{array}$ & $\begin{array}{l}3.20 \\
1.55\end{array}$ & $\begin{array}{r}100 \\
0.15\end{array}$ \\
\hline 021 & 5 & $\begin{array}{l}6.04 \\
0.33\end{array}$ & $\begin{array}{r}72.25 \\
0.68\end{array}$ & $\begin{array}{r}11.63 \\
0.12\end{array}$ & $\begin{array}{l}\mathbf{0 . 0 1} \\
0.02\end{array}$ & $\begin{array}{l}4.68 \\
0.19\end{array}$ & $\begin{array}{l}\mathbf{0 . 1 4} \\
0.02\end{array}$ & $\begin{array}{l}\mathbf{0 . 2 7} \\
0.04\end{array}$ & $\begin{array}{l}\mathbf{0 . 0 8} \\
0.61\end{array}$ & $\begin{array}{l}\mathbf{0 . 1 1} \\
0.06\end{array}$ & $\begin{array}{l}4.79 \\
0.15\end{array}$ & $\begin{array}{r}100 \\
0.05\end{array}$ \\
\hline 032 & 11 & $\begin{array}{l}4.13 \\
0.28\end{array}$ & $\begin{array}{r}75.64 \\
0.29\end{array}$ & $\begin{array}{r}13.44 \\
0.16\end{array}$ & $\begin{array}{l}\mathbf{0 . 0 3} \\
0.02\end{array}$ & $\begin{array}{l}4.94 \\
0.13\end{array}$ & $\begin{array}{l}\mathbf{0 . 0 1} \\
0.01\end{array}$ & $\begin{array}{l}0.48 \\
0.04\end{array}$ & $\begin{array}{r}-0.08 \\
0.45\end{array}$ & $\begin{array}{l}\mathbf{0 . 0 3} \\
0.05\end{array}$ & $\begin{array}{l}1.20 \\
0.12\end{array}$ & $\begin{array}{r}100 \\
0.43\end{array}$ \\
\hline 034 & 10 & $\begin{array}{l}4.17 \\
0.15\end{array}$ & $\begin{array}{r}75.63 \\
0.35\end{array}$ & $\begin{array}{r}13.32 \\
0.19\end{array}$ & $\begin{array}{l}0.03 \\
0.01\end{array}$ & $\begin{array}{l}4.91 \\
0.20\end{array}$ & $\begin{array}{l}\mathbf{0 . 0 3} \\
0.01\end{array}$ & $\begin{array}{l}0.46 \\
0.04\end{array}$ & $\begin{array}{l}\mathbf{0 . 1 0} \\
0.13\end{array}$ & $\begin{array}{l}0.05 \\
0.07\end{array}$ & $\begin{array}{l}1.25 \\
0.14\end{array}$ & $\begin{array}{r}100 \\
0.06\end{array}$ \\
\hline 041 & 10 & $\begin{array}{l}4.10 \\
0.12\end{array}$ & $\begin{array}{r}75.39 \\
0.25\end{array}$ & $\begin{array}{r}13.56 \\
0.15\end{array}$ & $\begin{array}{l}\mathbf{0 . 0 4} \\
0.01\end{array}$ & $\begin{array}{l}5.04 \\
0.14\end{array}$ & $\begin{array}{l}\mathbf{0 . 0 2} \\
0.01\end{array}$ & $\begin{array}{l}\mathbf{0 . 5 2} \\
0.03\end{array}$ & $\begin{array}{l}\mathbf{0 . 1 1} \\
0.05\end{array}$ & $\begin{array}{l}\mathbf{0 . 0 4} \\
0.07\end{array}$ & $\begin{array}{l}1.17 \\
0.11\end{array}$ & $\begin{array}{r}100 \\
0.02\end{array}$ \\
\hline
\end{tabular}

(1) : number of single grain analyses for each sample.

Tab. 3 : Résultats des analyses géochimiques sur les verres de ponce.

(1) Nombre de grains analysés. Analyses WDS (service Camparis, Paris, France): microsonde Cameca SX100, faisceau défocalisé à $10 \mu$ m; tension 15 kv; intensité $6 \mathrm{nA}$; temps de comptage ca. $10 \mathrm{~s}$; Valeurs normalisées à $100 \%$.

Tab. 3: Results of the single grain geochemical analyses of glass. (1) Number of analysed grains. WDS analyses (Camparis, Paris, France): microprobe Cameca SX 100, $10 \mu \mathrm{m}$ defocalized beam; tension $15 \mathrm{kv}$; intensity $6 \mathrm{nA}$; count time ca. $10 \mathrm{~s}$; normalized data. 


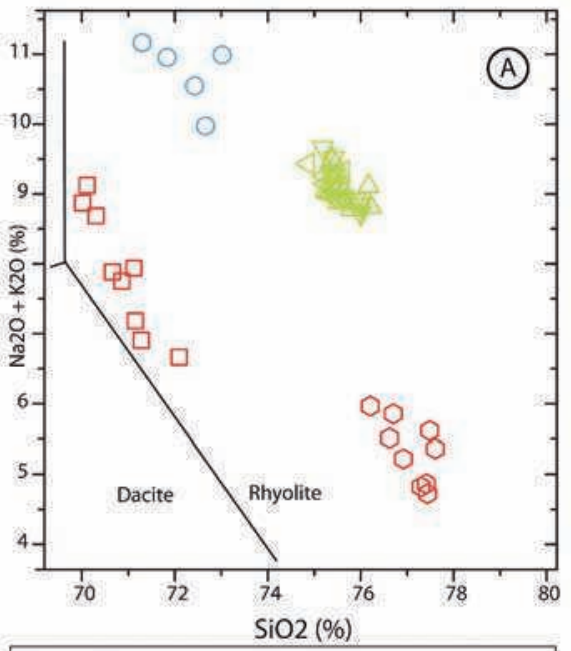

\begin{tabular}{|c|c|}
\hline Süphan: & Nemrut : \\
\hline$\triangle \operatorname{Van}-034(n=10)$ & $\square \operatorname{Van}-003(n=9)$ \\
\hline$\nabla \operatorname{Van}-032(n=11)$ & Van-002 $(n=9)$ \\
\hline $4 \operatorname{Van}-041(n=10)$ & $\begin{array}{l}\text { PYR-02: } \\
\quad \text { Van-021 }(n=5)\end{array}$ \\
\hline
\end{tabular}

$\mathrm{Ab}$
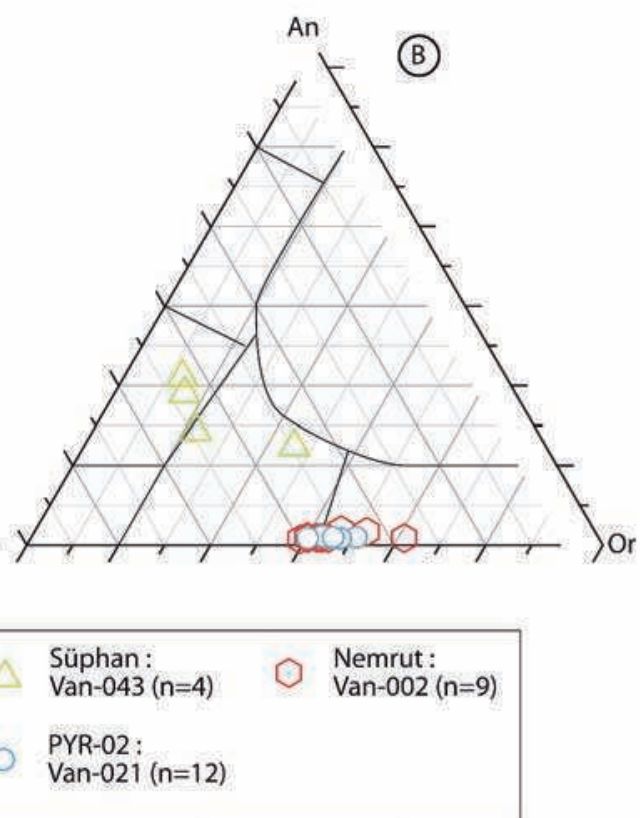

Fig. 6 : Géochimie de quelques pyroclastites provenant du pourtour du lac de Van. A : Verres de ponces ; B : Feldspaths.

Analyses WDS (service Camparis, Paris, France) : microsonde Cameca SX100, faisceau défocalisé à $10 \mu \mathrm{m}$; tension $15 \mathrm{kv}$; intensité 6nA ; temps de comptage ca. $10 \mathrm{~s}$; Valeurs normalisées à $100 \%$

Fig. 6: Geochemistry of some pyroclastites sampled around Van Lake. A: pumice glass; B: Feldspars. WDS analyses (Camparis, Paris, France): microprobe Cameca SX 100, $10 \mu \mathrm{m}$ defocalized beam; tension $15 \mathrm{kv}$; intensity 6nA; count time ca. $10 \mathrm{~s}$; normalized data.
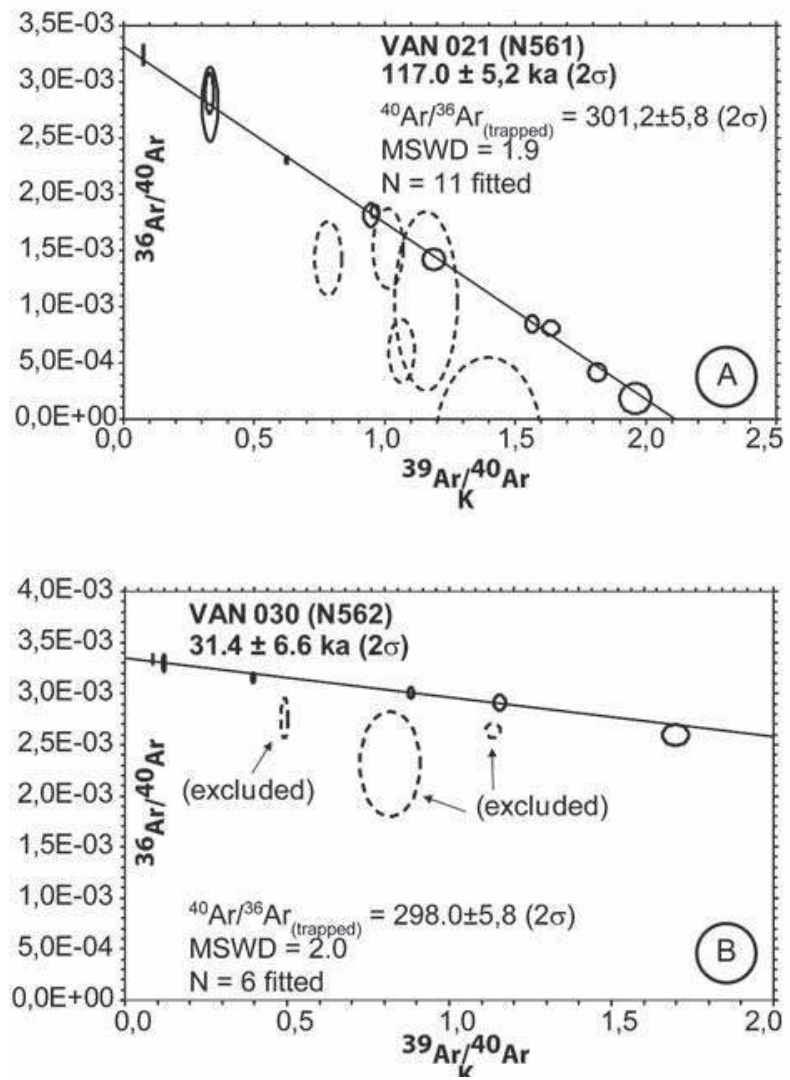

Fig. 7 : Âge Ar/Ar des feldspaths de deux téphras en connexion stratigraphique avec des dépôts lacustres.

7A: Echantillon VAN-021 provenant de la retombée plinienne de PYR-02. Son dépôt pré-date la transgression du cycle 1. 7B: Echantillon VAN-030 provenant d'une retombée plinienne localisée à l'est du lac, dont le dépôt pré-date la transgression du cycle 2.

Fig. 7: Ar/Ar age of feldspars from two tephra deposits in connexion with lake deposits. 7A: VAN-021 sample (PYR-02 Plinian fall). This deposit predates cycle 1 transgression. $7 B$ : VAN-030 sample, from the eastern part of the lake. This deposit predates cycle 2 transgression. les résultats concernant l'ensemble du lac de Van sont publiés par Kuzucuoğlu et al. (2010) et par Christol et al. (2010).

\section{4 - LES PYROCLASTITES}

Les logs stratigraphiques sont présentés sur la figure 8 . La figure 9 est une coupe synthétique localisée à l'aval de la vallée de Kotum, dessinée grâce aux nombreuses coupes révélées par une carrière. L'ensemble des logs et des coupes est localisé sur la figure 2. L'étude détaillée des pyroclastites présentes dans les trois vallées de Kotum, Küçüksu et du Güzeldere amène à différencier six «ensembles» de pyroclastites. Ces six «ensembles» pyroclastiques correspondent à: i) un téphra unique (PYR-03), ii) une pyroclastite présentant d'importants changements latéraux et verticaux de faciès (PYR-01 et PYR-05), ou iii) des pyroclastites différentes mises en place lors d'un cycle éruptif unique.

\section{1 - PYR-01}

Cet ensemble que nous appelons également «ignimbrite d'Obuz» du nom du village (à proximité de la vallée de la Küçüksu) à côté duquel les affleurements sont les plus épais, présente deux faciès distincts d'écoulements cendro-ponceux de couleur noire, admettant de grosses ponces également noires atteignant jusqu'à $60 \mathrm{~cm}$ de grand axe. Ces ponces ont une chimie trachytique (fig. 4 et 5 ). Elles sont très vésiculées, présentant des vésicules flexueuses, et très porphyriques, avec des feldspaths automorphes de taille millimétrique. On observe également de nombreux lithiques, plus nombreux à la base de cet 

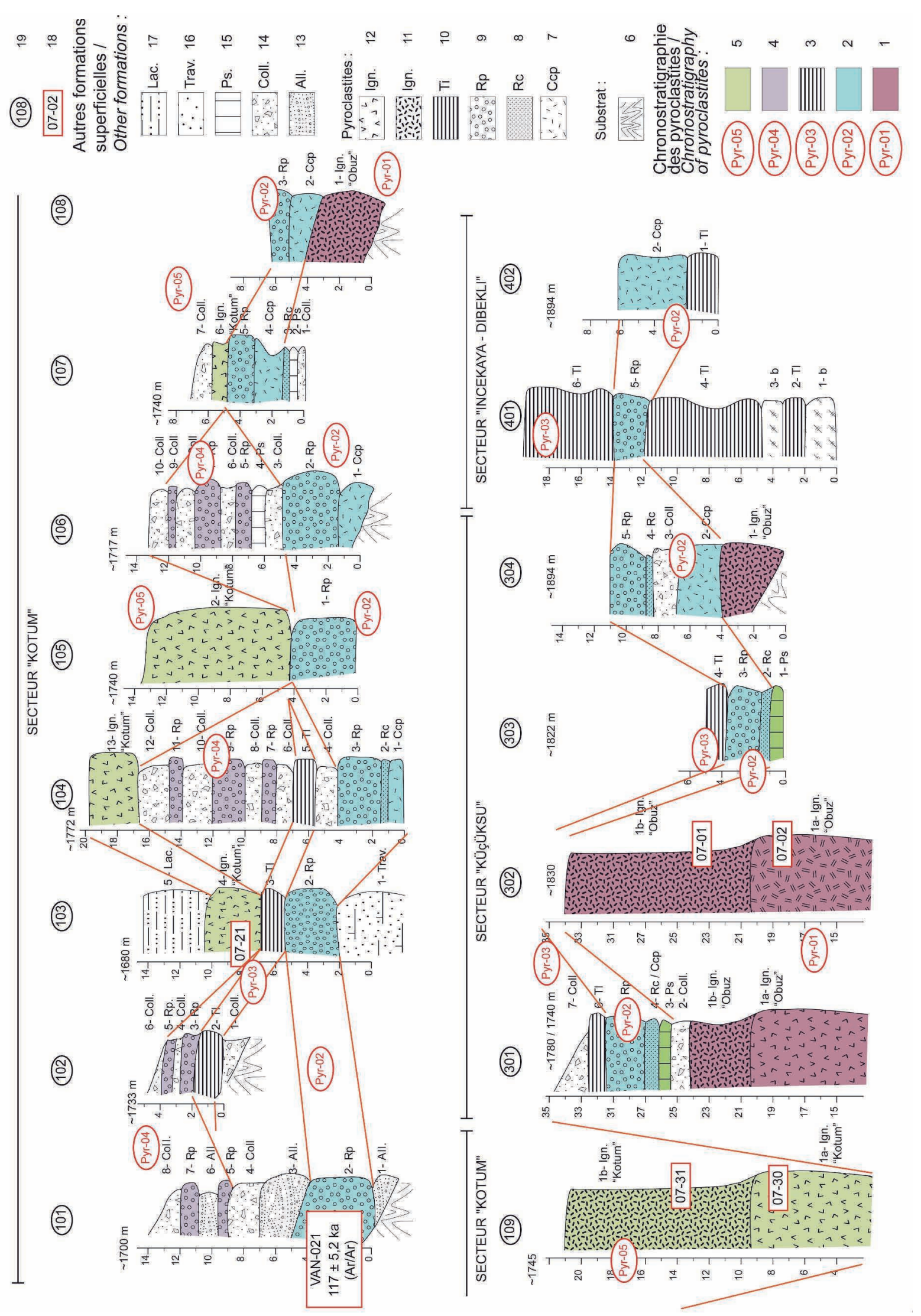

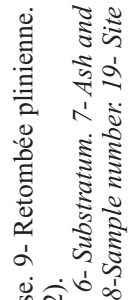
क्षें हु

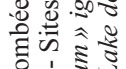
象行 क s: क्ष्य tót 要这

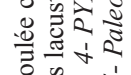

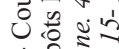
놀 대을 कि 施

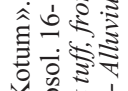
.0. क्ष 的宁 娄 50

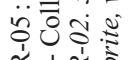
和高 的突方 ํํㄹ 究

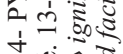
㺃 政: 证 壳的

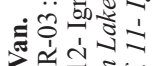
ช 光的焉 ㄴ. 产 产 三ิ 今.

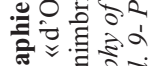
50. है 든 t.

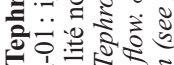

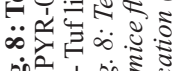

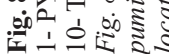


ensemble, qui montre des enclaves basaltiques atteignant $10 \mathrm{~cm}$ de grand axe.

La base de cet ensemble est indurée et présente des fiammes, alors que les faciès sommitaux sont non cohérents. Aucune autre formation ne vient jamais s'intercaler entre ces deux faciès, déposés lors du même épisode éruptif.

Cet ensemble PYR-01 est visible dans l'ensemble de la vallée de la Küçüksu et de ses affluents (notamment à leur entrée). Dans le secteur du village d'Obuz (coupes $n^{\circ}$ 302 et 301), elle dépasse la trentaine de mètres d'épaisseur, remplissant la vallée de la Küçüksu. Nous avons pu également l'observer sur les versants au niveau du village de Yoncabası, à proximité du village de Küçüksu $\left(n^{\circ} 108\right)$, ainsi que dans la vallée du Güzeldere ( $\left.n^{\circ} 201\right)$. En revanche, «l'ignimbrite d'Obuz» est absente de la vallée de Kotum.

\section{2 - PYR-02}

Cet ensemble correspond à un écoulement cendroponceux non cohérent, la plupart du temps difficilement visible (affleurements altérés), surmonté par une petite retombée cendreuse (10 cm d'épaisseur) puis par une retombée plinienne. Cette dernière affleure sur une grande partie du terrain (fig. 8). Le faciès dominant des ponces montre des vésicules inorganisées, avec cependant quelques ponces à vésicules parallèles. Les ponces sont microphyriques, comportant des feldspaths. La taille des ponces varie selon les affleurements : les plus grosses (jusqu'à $8 \mathrm{~cm}$ ) s'observent dans le secteur aval de la vallée de Kotum (coupe 101) et à proximité du village de Dalda; ailleurs, elles ne dépassent pas $5 \mathrm{~cm}$. De même la quantité des lithiques ainsi que leur taille sont variables, mais d'une manière générale cette quantité reste faible, parfois nulle, et les lithiques ont une taille ne dépassant pas quelques millimètres.

Au site 101 (fig. 2 et 8), les feldspaths de la retombée ponceuse (échantillon Van-021) ont été datés par $\mathrm{Ar} / \mathrm{Ar}$ et ont livré un âge de $117+/-$ 5,2 ka (fig. 7A).

\section{3 - PYR-03}

Cet ensemble correspond à une seule retombée grisnoire à noire, finement litée, qui constitue un horizonrepère sur une grande partie du secteur où elle affleure sur les versants où sur les sommets de terrasses dans la vallée de la Küçüksu et de Kotum : ce tuf noir recouvre les pyroclastites précédentes (PYR-02). Il est possible de suivre cette pyroclastite à partir de son point d'émission, le cône de tuf d'Incekaya, le long des vallées de la Küçüksu puis de Kotum où elle surplombe de $c a 90 \mathrm{~m}$ l'embouchure actuelle de la rivière dans le lac. A partir d'Incekaya, son épaisseur diminue, atteignant encore 1,5 $\mathrm{m}$ dans la coupe 102. Surtout, la granulométrie décroît: à proximité d'Incekaya, il s'agit de lapilli grossiers, alors qu'en position distale (coupe 102), elle est formée de fins lits de cendres avec de rares lapilli.

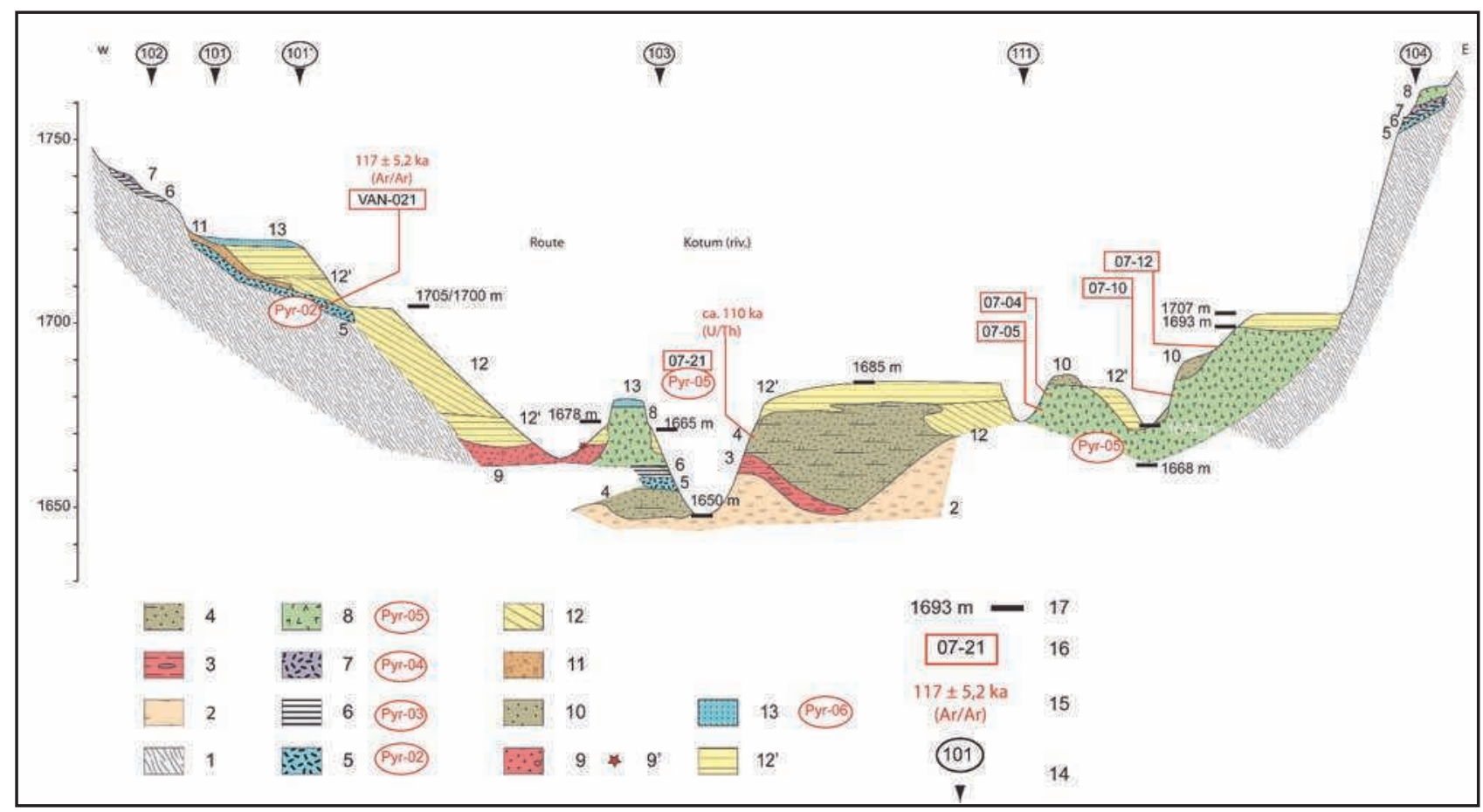

Fig. 9 : Pyroclastites et dépôts lacustres à l'aval de la vallée de Kotum.

1- Substrat. 2- Dépôts fluvio-lacustres. 3- Alluvions grossières. 4- Travertin. 5- Retombée plinienne (ensemble PYR-02). 6- Tuf noir lité (PYR-03). 7- Retombée plinienne (PYR-04). 8- Ignimbrite de Kotum (PYR-05). 9- Alluvions grossières remaniant 1'ignimbrite grise. 9'- fossile de Bos découvert en 2006. 10- Travertin sommital. 11- Dépôts de versant. 12- Formations lacustres (foreset). 12'- Formations lacustres (bottomset et shorefaces). 13- Retombée cendreuse (PYR-06). 14- Localisation des logs de la figure 8. 15- Âges radiométriques et méthode de datation. 16- Echantillons. 17- Altitudes mesurées au DGPS.

Fig. 9: Pyroclastites and lake deposits in the lower reach of Kotum river valley. 1-Substratum. 2- Fluvial and lake deposits. 3- Coarse alluvium. 4- Travertin. 5- Plinian fall (PYR-02). 6- Well bedded black tuff (PYR-03). 7- Plinian fall (PYR-04). 8- Kotum Ignimbrite (PYR-05). 9- Coarse alluviums reworking Kotum ignimbrite. 9'- Bos fossil discovered in 2006. 10-Upper travertin. 11- Slope deposits. 12-Lake deposits (foreset). 12'- Lake deposits (bottomset and shorefaces). 13-Ash fall (PYR-06). 14-Location of fig. 8 logs. 15-Radiomometric dating and used method. 16- Samples. 17- Elevation measured using a DGPS. 
Cette formation arme les replats supérieurs des vallées de la Küçüksu $(c a+30 \mathrm{~m})$ et de Kotum $(c a$ $+90 / 100 \mathrm{~m}$ ).

\section{4 - PYR-04}

Cet ensemble est formé d'une série de 3 retombées ponceuses, uniquement visibles dans la vallée de Kotum (coupes 101, 102, 104 et 106). Les ponces, dont la taille ne dépasse pas $2 \mathrm{~cm}$, sont peu vacuolaires, massives et riches en feldspaths. Les lithiques sont abondants, constitués de rhyolites et de diverses laves. Au sommet de chacune de ces retombées, des faciès de remaniement sont soulignés par l'enrichissement en matrice brune et par l'altération des ponces. Ces caractéristiques suggèrent des répits dans l'activité volcanique entre chacune des éruptions pliniennes formant PYR-04.

\section{5 - PYR-05}

Cet ensemble comprend une série d'écoulements pyroclastiques dont certains faciès sont indurés et que nous appelons ici «l'ignimbrite de Kotum » puisqu'elle remplit partiellement la vallée du même nom où elle atteint au maximum une soixante de mètres d'épaisseur. Les faciès indurés présentent des fiammes ponceux assez rares, et de nombreuses enclaves centimétriques d'obsidienne, de basalte et d'andésite. Les faciès non-indurés sont généralement enrichis en ponces.

A l'aval du village de Küçüksu, la rivière de Kotum incise cette ignimbrite dans laquelle elle décrit des méandres encaissés. Le replat inférieur observé dans cette vallée $(+15$ à $+30 \mathrm{~m})$ correspond au toit de cette ignimbrite (fig. 3C). Plus en aval, la rivière s'encaisse encore, mais dans des travertins, puis dans des dépôts lacustres surmontant à la fois les travertins et l'ignimbrite (fig. 9).

En amont du village de Küçüksu, l'ignimbrite a pénétré la vallée du Güzeldere (fig. 3B) dont elle forme le plancher et où elle est emboîté dans «l'ignimbrite d'Obuz» (PYR-01, cf. par exemple coupe $\mathrm{n}^{\circ}$ 201). Elle disparaît rapidement de la vallée du Güzeldere peu après le seuil topographique séparant le bassin-versant du lac de Van de celui du Tigre. En revanche, elle ne semble pas avoir pénétré dans la vallée de la Küçüksu où nous ne l'avons pas retrouvée.

\section{6 - PYR-06}

Les pyroclastites qui nappent les versants des trois vallées du secteur étudié sont généralement recouvertes par des dépôts colluviaux. Cependant, à l'aval de la vallée de Kotum, elles sont recouvertes par les dépôts lacustres associés à la transgression C1" du cycle 1. Quelques coupes permettent de constater que ces formations lacustres ou de pente sont à leur tour recouvertes par une retombée cendreuse blanche épaisse de 5 à $10 \mathrm{~cm}$, témoin de la dernière activité volcanique enregistrée dans le secteur. L'analyse géochimique et la caractérisation de ces produits terminaux sont en cours.

\section{5 - DISCUSSION ET CONCLUSION}

La discussion porte sur: i) l'origine des pyroclastites; ii) la succession stratigraphique et chronologique de ces produits volcaniques; iii) les implications morphologiques en terme de paléo-drainage et d'impact dans l'hydrographie régionale; et iv) l'implication de l'activité volcanique dans les variations du niveau du lac.

\section{ORIGINE DES PYROCLASTITES}

S'il n'est pas toujours possible de les suivre jusqu'au volcan source, il semble assez certain que les pyroclastites PYR-01, 02, 04 et 05 proviennent du Nemrut, alors que PYR-03 provient du cône de tuf d'Incekaya. L'origine de PYR-06 n'est pas encore connue.

«L'ignimbrite d'Obuz» (PYR-01) remplit essentiellement la vallée de la Küçüksu et celles de ses affluents, avec une épaisseur maximale un peu en amont du village d'Obuz. On l'observe également en couverture de versant dans la vallée du Güzeldere, y compris en aval du seuil topographique actuel; en revanche, elle est absente de la vallée de la Kotum.

Les analyses géochimiques (fig. 4 et 5) montrent que les trois échantillons provenant de cette unité forment un groupe relativement homogène. La signature géochimique de ces échantillons est proche de l'échantillon 07-46 qui provient de l'ignimbrite qui remplit la vallée de Bitlis au pied du Nemrut. Cette dernière est, selon Çukbuçu (2008), l'ignimbrite principale du Nemrut, de géochimie trachytique, associée à la phase d'effondrement de la caldeira sommitale. En outre, les caractéristiques pétrographiques de cette ignimbrite «du Nemrut» sont les mêmes que celles observées et analysées dans la coulée cendroponceuse noire (PYR-01): juvéniles composés de ponces sombres à noires, fiammes, lithiques variés provenant $\mathrm{du}$ substrat et de l'activité volcanique antérieure, faciès plus ou moins indurés (Çubukçu, 2008).

Concernant les ponces de l'ensemble PYR-02, les analyses géochimiques sont en cours. La diminution de la taille des ponces ainsi que celle des lithiques vers la partie orientale de notre secteur ainsi que, dans une moindre mesure, la diminution des épaisseurs de la retombée ponceuse, suggèrent que ces produits ont été émis par le Nemrut. Les premières analyses géochimiques à la microsonde dont nous disposons montrent: i) que l'échantillon Van-021 correspondant à la retombée ponceuse de PYR-02 a une signature géochimique différente des produits analysés provenant du Süphan (fig. 6A); et ii) qu'il contient des feldspaths dont la composition géochimique est identique à ceux provenant d'un échantillon que nous avons prélevé sur les flancs du Nemrut (fig. 6B).

L'origine du téphra PYR-03 ne pose pas de difficulté, puisque dans l'ensemble de notre secteur d'étude, ce tuf lité noir constitue un horizon repère unique, qu'il est possible de suivre jusqu'au cône de tuf d'Incekaya qui en constitue la source.

Les retombées ponceuses formant l'ensemble PYR-04 ne sont visibles que dans la partie nord-ouest de notre 
secteur. Les analyses géochimiques sont en cours. Cependant la répartition des affleurements suggère fortement qu'elles ont été émises par le Nemrut.

Enfin, l'ensemble PYR-05 correspond aux différents faciès de «l'ignimbrite» de Kotum qui affleure essentiellement dans la vallée du même nom, ainsi que dans la vallée du Güzeldere où son toit correspond au seuil potentiel de déversement actuel du lac de Van. Là encore, la disposition des affleurements et l'absence d'autre volcan ayant pu émettre de telles pyroclastites, suggèrent que cet ensemble a été émis par le Nemrut.

Selon Çubukçu (2008) une seconde ignimbrite est associée à l'effondrement de la caldeira: l'ignimbrite de «Kantaşı». L'ignimbrite «du Nemrut» et celle de «Kantaş1» seraient séparés par une activité plinienne qui pourrait correspondre à nos pyroclastites PYR-04. La description faite par Çubukçu (2008) de l'ignimbrite de «Kantaş1» indique des similitudes avec l'ignimbrite de Kotum, mais il ne publie aucune analyse géochimique sur roche totale avec lesquelles nous pourrions comparer nos résultats.

Enfin, en l'attente de nouvelles analyses géochimiques, l'origine de PYR-06 (retombée cendreuse supérieure) reste inconnue. Celle-ci joue cependant un rôle secondaire dans la téphrostratigraphie régionale et, surtout, dans l'évolution des morphologies.

\section{CHRONOLOGIE DE LA MISE EN PLACE DES PYRO-} CLASTITES

Il est possible de reconnaître un certain nombre de hiatus dans la succession des dépôts des pyroclastites PYR-01 à -06 :

- On observe un petit paléosol peu organique à la base de PYR-02 dans les coupes 107, 301 et 303 (fig. 2 et 8). Dans la coupe 301, ce paléosol est développé sur des colluvions qui reposent sur «l'ignimbrite d'Obuz» (PYR-01). Colluvions et paléosol suggèrent un arrêt de l'activité volcanique entre le dépôt des ensembles PYR-01 et PYR-02.

- Dans le secteur de la Küçüksu, le tuf noir lité (PYR03) repose soit sur la retombée plinienne de PYR-02 (coupe 301 et 303), soit sur des alluvions qui ellesmêmes reposent sur «l'ignimbrite d'Obuz» (PYR-01, cf. fig. 3A). Cette disposition témoigne de l'incision de PYR-01 et d'un remblaiement alluvial antérieur au dépôt de PYR-03.

- Cette incision est également visible dans le secteur du Güzeldere (fig. 3B). Là, «l'ignimbrite de Kotum» (PYR05) est emboîtée dans «l'ignimbrite d'Obuz» (PYR-01) qui, dans cette vallée, n'affleure plus que sous forme de lambeaux nappant certains versants, alors que le fond plat de la vallée est constitué par le toit de «l'ignimbrite de Kotum ». Cet emboîtement témoigne, là encore, d'une phase d'incision postérieure au dépôt de PYR-01.

On observe donc une phase d'altération et de pédogénéisation sur les versants et une phase d'incision suivie d'un remblaiement alluvial dans toutes les vallées du secteur à l'exception de celle de Kotum où PYR-01 n'affleure pas et où elle a peut-être été entièrement déblayée par la paléo-Kotum. Il est possible que cette évolution différenciée des versants et des fonds de vallée soit cependant contemporaine. Elle signe un arrêt de l'activité volcanique postérieure au dépôt de «l'ignimbrite d'Obuz» (PYR-01).

La suite de l'accumulation de pyroclastites, jusqu'à PYR-05 semble plus continue, sans hiatus majeur. Les coupes montrent parfois des colluvions séparant certains téphras, mais il peut s'agir d'un remaniement qui suit très rapidement le dépôt d'une pyroclastite sur des versants en pente. Ces «colluvions» ne semblent donc pas indiquer un arrêt majeur de l'activité volcanique entre PYR-03 et PYR-05.

- Enfin, on observe un hiatus entre PYR-05 et PYR-06, puisque cette dernière retombée cendreuse est toujours séparée des pyroclastites précédentes par des dépôts colluviaux (sites 101) ou lacustres (site 101'). Entre le dépôt de PYR-05 et de PYR-06, se produit donc la transgression lacustre $\mathrm{C} 1$ " et le dépôt des formations qui soustendent la terrasse d'accumulation T2.

\section{ÂGES RADIOMÉTRIQUES}

La datation radiométrique par Ar/Ar de 10 échantillons provenant de ces différentes pyroclastites est en cours. Pour le moment, nous ne disposons que de quelques jalons qui permettent de rapporter les pyroclastites PYR-01 à PYR-05 au Pléistocène moyen, aux alentours de $110 \mathrm{ka}$.

Les feldspaths de l'échantillon VAN-021, provenant de la retombée plinienne (PYR-02) du site 101, ont livré un âge $\mathrm{Ar} / \mathrm{Ar}$ de $117 \pm 5,2 \mathrm{ka}$. Cette date est cohérente avec l'âge du travertin, daté dans la carrière de Kotum de $c a 110 \mathrm{ka}$ (Kuzucuoğlu et al., 2010). Ces deux formations sont recouvertes par les dépôts lacustres de la transgression du cycle 1 .

La date de cette retombée plinienne signifie que «l'ignimbrite d'Obuz» (PYR-01), que nous assimilons à «l'ignimbrite du Nemrut» décrite par Çubukçu (2008), est antérieure à $c a 117 \mathrm{ka}$. Or, selon cet auteur, l'âge de «l'ignimbrite du Nemrut» serait compris entre 100 et $30 \mathrm{ka}$. En effet, l'âge K/Ar de trois dômes recouverts par cette ignimbrite est compris entre 83 et $99 \mathrm{ka}$, et les édifices les plus anciens recoupant l'ignimbrite sont datés de ca 30 ka (Çubukçu, 2008).

L'âge que nous avons obtenu sur PYR-02 vieillit donc d'au moins 15 ka l'âge proposé par Çubukçu (2008) pour «l'ignimbrite du Nemrut». En attendant les nouvelles dates sur les pyroclastites du secteur, nous ne sommes pas capables d'expliquer cette contradiction.

$\mathrm{Au} \mathrm{vu} \mathrm{de} \mathrm{nos} \mathrm{résultats,} \mathrm{il} \mathrm{paraît} \mathrm{cependant} \mathrm{très}$ probable que le laps de temps qui sépare l'émission de «l'ignimbrite d'Obuz» (PYR-01) de celle de «l'ignimbrite de Kotum»(PYR-05) immédiatement antérieure à la transgression C1", semble avoir été assez court, malgré le hiatus marqué par la pédogènèse des versants et le dépôt d'alluvions grossières dans le bassin de la Küçüksu. 
IMPLICATIONS GÉOMORPHOLOGIQUES DE L'ACTIVITÉ VOLCANIQUE DANS LE RÉSEAU HYDROGRAPHIQUE

L'activité volcanique enregistrée au sud-ouest du lac de Van a fortement perturbé le réseau hydrographique en remplissant et fossilisant des paléo-vallées, et en séparant certaines vallées de leur bassin-versant amont. L'étude des paléo-topographies fossilisées par les pyroclastites permet de reconstituer l'évolution du drainage au sudouest du lac de Van (fig. 10).

Phase 1. Trois larges vallées étaient probablement raccordées au secteur sud-ouest du bassin, aujourd'hui occupé par le lac: la vallée de Bitlis, celle de Muş et la paléovallée Küçüksu-Güzeldere. Toutes trois se dirigeaient vers le bassin du Tigre (fig. 10A). Il est probable qu'au moins l'une des trois vallées servait d'exutoire au lac. La paléovallée de Küçüksu-Güzeldere devait probablement prendre naissance dans la baie de Düzcealan, à cette époque où les volcans de Dibekli-Incekaya n'existaient pas encore.

Phase 2 (fig. 10B). Lors de sa mise en place, «l'ignimbrite d'Obuz» (PYR-01 = « ignimbrite du Nemrut» selon Çubukçu, 2008) a recouvert l'ensemble des flancs du Nemrut. Au sud, elle est venue butter sur les contreforts septentrionaux du massif de Bitlis. Elle a, ce faisant, pénétré et fossilisé les deux principales vallées: celle de Bitlis et de Küçuksu-Güzeldere. Son dépôt est à l'origine de deux seuils topographiques: le premier à $c a .1785 \mathrm{~m}$, bloquant le paléo-drainage vers Muş et Bitlis; le second à $c a .1780 \mathrm{~m}$ (faciès meuble de PYR-01) et $c a .1750 \mathrm{~m}$ (faciès induré) bloquant la paléovallée de Küçuksu-Güzeldere. Le lac de Van s'est alors trouvé en situation endoréique, ce qui est responsable de la transgression $\mathrm{Cl}$ '.

Phase 3 (fig. 10C). L'érosion des faciès «meubles» de «l'ignimbrite d'Obuz» (PYR-01) qui affleurent essentiellement dans la vallée de la Küçüksu permet le rétablissement du paléo-drainage Küçuksu-Güzeldere, expliquant la régression enregistrée entre $\mathrm{C} 1$ ' et $\mathrm{C} 1$ ". Cette incision est marquée par: i) le déblaiement total de PYR-01 de la vallée de Kotum; ii) l'emboîtement des pyroclastites postérieures dans PYR-01 dans les vallées de Küçüksu et du Güzeldere); et par iii) le remblaiement alluvial observé dans la vallée de la Küçüksu.

Cette phase d'incision et de remblaiement peut avoir été d'autant plus rapide que dans la vallée de la Küçüksu, le faciès de l'ignimbrite d'Obuz est essentiellement peu cohérent. En outre, cette phase est située entre le dépôt de PYR-01 et celui de PYR-03 et correspond donc approximativement au dépôt de PYR-02, daté de 117 ka.

Phase 4 (fig. 10D). L'activité volcanique dans le secteur Incekaya-Dibekli et la mise en place du cône de tuf d'Incekaya est responsable du dépôt de PYR-03 qui fossilise les pyroclastites antérieures ainsi que les alluvions de fond de vallée. Cette activité volcanique entraine la fermeture de la paléovallée Küçuksu-Güzeldere qui se trouve séparée de son bassin-versant amont et de sa connexion avec le lac au niveau de la baie de Düzcelan. Cette nouvelle fermeture est responsable du début de la transgression $\mathrm{C} 1$ ".
Phase 5 (fig. 10E). «L'ignimbrite de Kotum» (PYR05) fossilise la vallée du même nom et une partie de la paléo-vallée Küçuksu-Güzeldere, en aval du village de Küçüksu. Le toit de cette ignimbrite forme un nouveau seuil topographique, aujourd'hui situé à $1736 \mathrm{~m}$. Ce dépôt contribue à la continuité de la transgression $\mathrm{C} 1$ ". Le lac de Van, devenu un lac terminal, est définitivement endoréique.

Phase 6. (fig. 10F). Suite au dépôt de «l'ignimbrite de Kotum» (PYR-05), le drainage est totalement désorganisé. L'incision régressive de la Kotum dans PYR-05 conduit à la capture de la Küçüksu par la petite rivière de la vallée de Kotum et donc à l'addition du haut-bassin versant de la Küçüksu au bassin-versant du lac.

En plus des implications relatives au paléodrainage régional, l'activité volcanique est également responsable de la plupart des morphologies de détail observées dans les vallées du secteur de Tatvan. Ainsi, les replats qui accidentent les vallées de la Küçüksu et de Kotum ne sont pas de simples terrasses alluviales ou lacustres, mais sont toujours associés à des pyroclastites. Dans la vallée de la Küçüksu, le replat observé à ca $30 \mathrm{~m}$ correspond partout à l'affleurement du tuf noir lité d'Incekaya (PYR-03), même quand celui-ci fossilise directement des alluvions. A l'aval de la vallée de Kotum, trois replats d'inégale importance sont situés à $c a+30 \mathrm{~m}, c a+70 \mathrm{~m}$ et $c a+90 / 100 \mathrm{~m}$. Le premier correspond à l'affleurement de l'ignimbrite de Kotum qui, encaissée en amont dans PYR-01 passe vers l'aval à des travertins qui la surmontent, puis à des dépôts lacustres de type bottomsets qui la recouvrent également. Il s'agit donc d'une terrasse d'érosion nivelant des formations pyroclastiques, travertineuses et lacustres, encaissées dans l'ensemble des formations antérieures (de PYR-01 à PYR-03). Le second replat ( $c a$ +70 ) est formé par la retombée ponceuse PYR-02 recouverte par des dépôts de versant puis des dépôts lacustres. Enfin le troisième replat $(c a+90 / 100 \mathrm{~m})$ correspond à l'affleurement du tuf noir lité (PYR-03).

\section{IMPLICATIONS DE L'ACTIVITÉ VOLCANIQUE DANS LES VARIATIONS DU NIVEAU DU LAC DE VAN}

La première transgression du lac $(\mathrm{C} 1)$ qui est responsable des plus hautes terrasses lacustres autour du lac, présente dans le détail une succession de deux hauts niveaux (C1' et C1', cf. Kuzucuoğlu et al., 2010) correspondant d'une part (C1') aux terrasses lacustres de la Zilan et de la Karasu dans la partie orientale du lac, et d'autre part aux dépôts transgressifs de Kotum (1725 m, $c a+80 \mathrm{~m}$ ), également préservés dans les secteurs amont des basses vallées de tous les grands affluents du pourtour du lac (Karasu, Engil, Zilan).

Cette succession établie par Kuzucuoğlu et al. (2010) correspond parfaitement avec la stratigraphie des pyroclastites dans le secteur de Tatvan.

Notre reconstruction montre que «l'ignimbrite d'Obuz» (PYR-01) a rempli les vallées de Bitlis et de la Küçüksu, dont l'une au moins servait d'exutoire au lac de Van. Il est possible qu'elle soit responsable de la trans- 


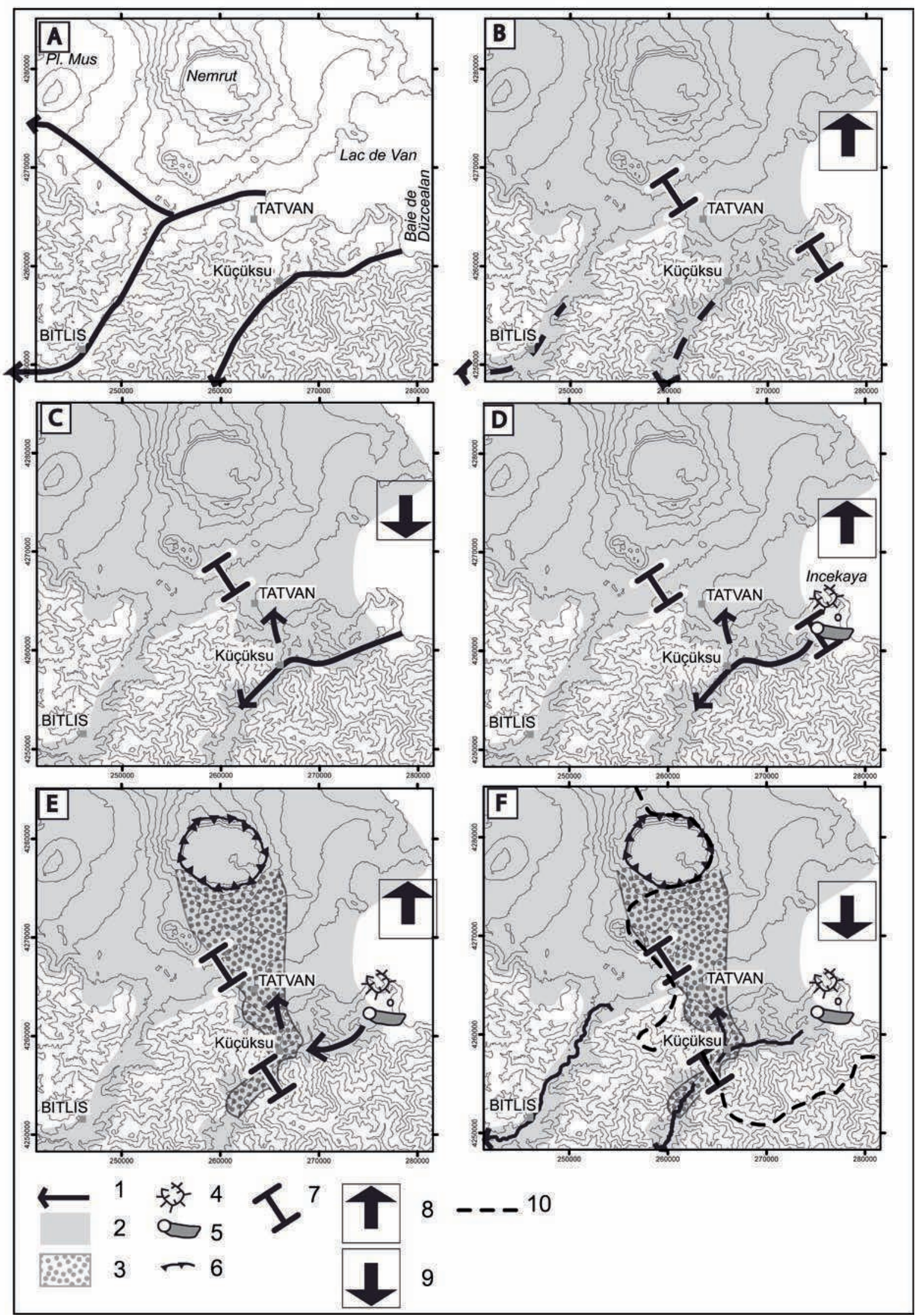

Fig. 10 : Evolution des paléogéographies et de la paléohydrographie, déterminées par les émissions successives de pyroclastites du Nemrut dans le secteur de Tatvan.

1- Drainage et paléo-drainage. 2- PYR-01, ignimbrite d'Obuz. 3- PYR-05, ignimbite de Kotum. 4- Cône de tuf (Incekaya). 5- Cône de scorie et coulée de lave associée. 6- Caldeira du Nemrut. 7- Barrages d'origine volcanique. 8- Transgression lacustre. 9- Régression lacustre. 10- Limite actuelle du bassin versant.

Fig. 10: Evolution of palaeogeography and palaeohydrology linked to Nemrut volcanic activity around Tatvan (SW of Lake Van). 1- Drainage and palaeo-drainage. 2- PYR-01, Obuz ignimbrite. 3- PYR-05, Kotum ignimbrite. 4- Tuff cone (Incekaya). 5- Scoria cone and associated lava flow. 6- Nemrut Caldera. 7-Volcanic dam. 8-Lake level transgression. 9- Lake level regression. 10- Present day drainage area. 
gression C1' décrite par Kuzucuoğlu et al. (2010). Dans le secteur étudié ici, aucun dépôt lacustre ne témoigne de cette première transgression: il est possible qu'ils aient été érodés et/ou fossilisés par l'évolution ultérieure.

L'érosion de cette première ignimbrite est d'autant plus facile que son faciès est souvent peu cohérent, dans les vallées de Kotum, Güzeldere et Küçüksu du moins. Alors que dans la vallée de Bitlis, il s'agit souvent de faciès très cohérent. Cette érosion pourrait expliquer la régression intermédiaire comprise entre C1' et C1" (Kuzucuoğlu et al., 2010).

Enfin, «l'ignimbrite de Kotum» (PYR-05) est recouverte par des bottomsets lacustres (site 103, fig. 8 et 9) qui témoignent de la transgression C1" (Kuzucuoğlu et al., 2010). Ces dépôts lacustres recouvrent la retombée ponceuse de PYR-01, datée de $117 \mathrm{ka}$, le travertin daté de $110 \mathrm{ka}$ et «l'ignimbrite de Kotum», postérieure aux précédents. Ce haut niveau du lac $\mathrm{C} 1$ ", qui forme tout autour du lac la terrasse de $1726 \mathrm{~m}(c a+80 \mathrm{~m})$ et qui correspond à un niveau du lac estimé à 1732/1 $735 \mathrm{~m}$ est donc postérieur à l'ignimbrite de Kotum.

\section{CONCLUSION}

Au total, l'activité volcanique a été responsable, aux alentours d'une période immédiatement antérieure ou centrée sur $c a 117 \mathrm{ka}$ : i) de la désorganisation du réseau hydrographique et ii) de la fermeture du lac de Van.

En effet, la mise en place des ignimbrites est responsable du remblaiement de la plupart des vallées situées à l'ouest du lac. En outre, les volcans monogéniques de Dibekli-Incekaya ont amputé la vallée de la Küçüksu d'une partie de son bassin-versant amont. L'activité volcanique explique donc les morphologies actuelles: vallées sur-calibrées, replats armés par des pyroclastites, etc.

De plus, les ignimbrites sont à l'origine de la fermeture du lac de Van qui s'est probablement effectuée en deux temps, correspondant à deux épisodes volcaniques responsables de la mise en place de deux ignimbrites et provoquant à chaque fois une transgression lacustre. La première transgression est corrélée à la mise en place de l'ignimbrite «d'Obuz» (PYR-01) antérieure à $117 \mathrm{ka}$; alors que la seconde est liée à l'ignimbrite «de Kotum» (PYR-05) postérieure à $c a 110 \mathrm{ka}$. Lors de ces transgressions, le dépôt des sédiments formant les terrasses de $c a+80 \mathrm{~m}$ et enregistrant un niveau du lac atteignant $c a 1732 \mathrm{~m}$, témoignent d'un bilan hydrique plus humide que l'actuel puisque ce dernier établit un niveau $\mathrm{du}$ lac relativement stable à 1646-1649 m. Cependant, nous pouvons affirmer que le déclenchement de ces deux transgressions rapprochées, à la fin du Pléistocène moyen, ne correspond pas uniquement à une variation du bilan hydrique, mais semblent d'abord répondre à l'activité volcanique du Nemrut qui a provoqué la fermeture d'une dépression occupée auparavant par un lac plus circonscrit qu'aujourd'hui dans la seule partie orientale du bassin.

\section{REMERCIEMENTS}

Les auteurs remercient les institutions qui ont soutenu le projet «ANOVAN» (2006-2008): le TÜBITAK (ÇAYDAK 105Y125; SOBAG 105Y127), le CNRS (ECLIPSE II et PICS), l'Université de Van (FED-B-10), le ministère français des Affaires étrangères (PHC) et l'ambassade de France à Ankara. Ils remercient également leurs laboratoires respectifs (UMR 6266, 8591, 1572 et 8586) qui ont participé au soutien financier nécessaire au fonctionnement du programme. Enfin, ils remercient les deux relecteurs, E. Defive et G. Vernet, dont les lectures attentives ont permis d'améliorer la clarté de cet article.

\section{RÉFÉRENCES BIBLIOGRAPHIQUES}

AYDAR E., GOURGAUD A., ULUSOY I., DIGEONNET F., LABAZUY P., SEN E., BAYHAN H., TURKER K., \& TOLLUOGLU A.U., 2003- Morphological analysis of active Mount Nemrut stratovolcano, eastern Turkey: evidences and possible impact areas of future eruption. Journal of Volcanology and Geothermal Research, 123, 301-312.

CHRISTOL A., KUZUCUOGLU C., FORT M., KARABIYIKOĞLU M., MOURALIS D., BRUNSTEIN D., DOG̈U A.-F., AKKÖPRÜ E., FONTUGNE M., \& ZORER H., 2008 - Apports de l'étude géomorphologique des terrasses fluvio-lacustres du lac de Van (Turquie) à la connaissance des paléoenvironnements en Anatolie orientale. In D. Galop, (ed.), Paysages et Environnements. De la reconstitution du passé aux modèles prospectifs. Annales littéraires, Série «Environnement, sociétés et archéologie», P.U. de FrancheComté, Besançon, 115-130.

CHRISTOL A., KUZUCUOĞLU C., FORT M., MOURALIS D., DOĞU A.-F., AKKÖPRÜ E., BRUNSTEIN D., FONTUGNE M., KARABIYIKOĞLU M., SCAILLET S., \& ZORER H., 2010 - Les indicateurs morphosédimentaires des variations de niveau lacustre au Pléistocène supérieur dans les terrasses autour du lac de Van (Turquie). Quaternaire, 21 (4), 443-458.

ÇUBUKÇU E., 2008 - Evolution pétrologique du strato-volcan Nemrut Dagi (Turquie): magmatisme hyperalcalin en domaine de collision. Thèse de Doctorat, Université Blaise Pascal, Clermont-Ferrand II, $221 \mathrm{p}$.

DEGENS E.T., \& KURTMANN F., 1978 - Geology of Lake Van. MTA Press, 169, Ankara, $158 \mathrm{p}$.

KARAKHANIAN A., DJRBASHIAN R., TRIFONOV V., PHILIP H., ARAKELIAN S., \& AVAGIAN A., 2002 - Holocene-historical volcanism and active faults as natural risk factors for Armenia and adjacent countries. Journal of Volcanology and Geothermal Research, 113, 319-344. KARAOĞLU Ö., ÖZDEMIR Y., TOLLUOGLU A.̈̈.,
KARABIYIKOĞLU M., KÖSE O., \& FROGER J.-L., 2005 Stratigraphy of the volcanic products around Nemrut Caldera: implications for reconstruction of the Caldera formation. Turkish Journal of Earth Sciences, 14, 123-124.

KEMPE S., LANDMANN G., \& MÜLLER G., 2002 - A floating varve chronology from the last glacial maximum terrace of Lake Van/Turkey. Zeitschrift für Geomorphologie Supplementbände, 126, 97-114.

KUZUCUOĞLU C., CHRISTOL A., MOURALIS D., DOĞU A.-F., AKKÖPRÜ E., FORT M., BRUNSTEIN D., ZORER H., FONTUGNE M., KARABIYIKOĞLU M., SCAILLET S., REYSS J.-L., \& GUILLOU H., 2010 - Formation of the Upper Pleistocene terraces of Lake Van (Turkey). Journal of Quaternay Sciences, 25 (7), 1124-1137.

LE MAITRE R.W. (ed.), 2002 - Igneous Rocks: A Classification and Glossary of Terms, Recommendations of the International Union of Geological Sciences, Subcommission of the Systematics of Igneous Rocks. Cambridge University Press, xvi +236 p.

MOURALIS D., GUILLOU H., SCAILLET S., KUZUCUOĞLU C., CHRISTOL A., AKKÖPRÜ E., FONTUGNE M., DOĞU A.-F., ZORER H., \& KARABIYIKOGLU M., 2007 - Les téphras interstratifiés dans les terrasses lacustres du lac de Van (Turquie): marqueurs chronostratigraphiques et identifications des volcans sources (Nemrut, Süphan, etc.). Résultats préliminaires. Colloque CNRS-ECLIPSE, Paris, 15-16 octobre 2007. 
MOURALIS D., GUILLOU H., SCAILLET S., KUZUCUOGLU C., CHRISTOL A., AKKÖPRÜ E., FONTUGNE M., DOĞU A.-F. ZORER H., \& KARABIYIKOĞLU M., 2008 - L'activité volcanique sur le pourtour du lac de Van (Turquie): impacts morphologiques et utilisation comme marqueur chronostratigraphique. Colloque AFEQ-INQUA Q6, Montpellier, 26-28 février 2008.

OSWALD F., 1912 - Armenien. Carl Winter, Heidelberg, 40 p.

ÖZDEMIR Y., KARAOĞLU Ö., TOLLUOĞLU A.Ü., \& GÜLEÇ N., 2006 - Volcanostratigraphy and petrogenesis of the Nemrut stratovolcano (East Anatolian High Plateau): The most recent postcollisional volcanism in Türkey. Chemical Geology, 226, 189-211.

SCAILLET S., 1996 - Excess 40Ar transport scale and mechanism in high-pressure phengites: A case study from an eclogitized metabasite of the Dora-Maira nappe, western Alps. Geochimica et Cosmochimica Acta, 60, 1075-1090
SCAILLET S., 2000 - Numerical error analysis in 40Ar/39Ar dating. Chemical Geolology, 162, 269-298.

ULUSOY İ., LABAZUY P., AYDAR E., ERSOY O., \& CUBUKCU E., 2008 - Structure of the Nemrut caldera (Eastern Anatolia, Turkey) and associated hydrothermal fluid circulation. Journal of Volcanology and Geothermal research, 74, 269-283.

VALETON I., 1978 - A morphological and petrological study of the terraces around Lake Van, Turkey. In E. Degens \& F. Kurtmann (ed.), Geology of Lake Van. MTA Press, Ankara, 64-80.

YILMAZ Y., GÜNER Y., \& SAROĞLU F., 1998 - Geology of the Quaternary volcanic centres of the east Anatolia. Journal of Volcanology and Geothermal Research, 85, 173-210. 